\title{
Semiclassical many-mode Floquet theory. IV. Coherent population trapping and SU(3) dynamical evolution of dissipative three-level systems in intense bichromatic fields
}

\author{
Tak-San Ho and Shih-I Chu \\ Department of Chemistry, University of Kansas, Lawrence, Kansas 66045
}

(Received 5 December 1984)

\begin{abstract}
The many-mode Floquet theory (MMFT) recently developed by authors is extended to incorporate the irreversible damping mechanisms for the nonperturbative treatment of the dynamical evolution of dissipative three-level systems at two-photon or multiphoton coherent resonance trapping conditions induced by two strong linearly polarized monochromatic fields. It has been recently shown by several workers that under the rotating-wave approximation (RWA), population may be permanently trapped in the three-level system if the coherent monochromatic fields are exactly two-photon resonant with the initial and final states, decoupled from the intermediate decaying level. In practice, the inclusion of the non-RWA terms necessarily modifies the resonant trapping conditions and behavior. In this paper we extend the generalized Van Vleck (GVV) nearly degenerate perturbation theory to an analytical treatment of the non-Hermitian two-mode Floquet Hamiltonian. This reduces the infinite-dimensional time-independent non-Hermitian Floquet Hamiltonian to a $3 \times 3$ effective Hamiltonian, from which essential properties of the coherent population-trapping behavior as well as the dynamical evolution of the dissipative $\mathbf{S U}(3)$ coherence vector $\mathbf{S}(t)$ can be readily obtained and expressed in terms of only three complex quasienergy eigenvalues and eigenvectors. The MMFT-GVV studies show that the RWA two-photon resonant trapping condition is substantially modified by the effects of non-RWA terms, and that the system can be "quasitrapped" for only a finite amount of time characterized by a small imaginary energy (width) associated with a coherent superposition state of the initial and final levels. Furthermore, it is found that the initially eightdimensional coherence vector $\mathbf{S}(t)$ evolves predominantly to a one-dimensional scalar at the twophoton or multiphoton resonant quasitrapping conditions. Detailed results and pictorial representations of the population trapping and SU(3) dissipative dynamical evolution are presented.
\end{abstract}

\section{INTRODUCTION}

Recent developments in semiclassical Floquet theories have provided new insights and practical nonperturbative methods for the investigation of intense-field multiphoton excitation (MPE), ionization (MPI), and dissociation (MPD) processes. $^{1}$ In particular, the development of the many-mode Floquet theory ${ }^{2-4}$ (MMFT) allows for the first time exact reformulation of the time-dependent problem of the interaction of finite-level systems with polychromatic fields as an equivalent time-independent infinite-dimensional eigenvalue problem. In a previous paper $^{2}$ (hereafter called paper I), the authors have developed a general formulation of the MMFT, which is an exact extension of Shirley's single-mode Floquet theory. ${ }^{5}$ In a subsequent paper (paper II), ${ }^{3}$ detailed nonlinear responses of two-level ( $\operatorname{spin}-\frac{1}{2}$ ) systems under the influence of intense bichromatic fields are examined, and useful analytic expressions for MPE transition probabilities, bichromatic Bloch-Siegert shifts, and resonance widths, etc., are obtained. In a more recent paper (paper III) ${ }^{4}$ the authors further exploit, both analytically and pictorially, the vivid geometry and dynamical evolution of the eight-dimensional $\mathbf{S U}(3)$ coherent vector ${ }^{6,7} \mathbf{S}(t)$ characterizing the interaction of dissipationless three-level systems with intense bichromatic fields. In this paper, we extend the MMFT to the study of the dynamical behavior of $N$-level systems with dissipative couplings under the influence of strong bichromatic fields. The dissipative terms are the realization of the irreversible loss processes such as collisional relaxations and spontaneous decays out of the system, etc. Particular attention will be addressed to the investigation of the phenomenon of coherent population trapping as well as the geometrical and time evolution of the SU(3) coherent vector of dissipating three-level systems.

The subject of coherent population trapping in threelevel systems has received much attention recently both theoretically $^{8-10}$ and experimentally. ${ }^{11,12}$ It has been shown that ${ }^{10}$ under the rotating-wave approximation (RWA), population may be trapped in the three-level system if the coherent monochromatic driving fields are exactly two-photon resonant with the initial and final states. Population trapping is generated by a coherent superposition of the initial and final states, decoupled from the intermediate decaying level. Such coherent superposition states are characterized by real dress states (or quasienergy states) with zero widths and are immune to further photoexcitation. The deep "dark resonances"11,12 or the narrow coherence minima observed in the Raman absorption line shape or the total fluorescence signal as a function of detuning are thought to be manifestations of the coherent trapping of the population. These narrow trapping holes have been extensively studied in view of possible applications in high-resolution spectroscopy and for potential time and frequency standards. ${ }^{13}$ The effects of finite field bandwidths, spontaneous emissions and/or correlation between fields on coherent population trapping 
have also been studied and it has been found that the trapping can be either destroyed, preserved, or even enhanced. ${ }^{10}$ What is not known yet are the effects of non-RWA terms on the trapping, that is, how and to what extend the coherent trapping conditions will be modified when the RWA model breaks down in intense fields. This is one of the main questions we shall address in this paper.

There are in general two ways of implementing the damping mechanisms in the Floquet matrix formalism. The first method is to incorporate the bound-tocontinuum couplings self-consistently and generate the dissipation rates in an a priori way. This has been accomplished previously, in the case of interaction with single monochromatic field, by the so-called non-Hermitian Floquet theory and complex-quasienergy methods for the treatment of intense-field MPI of atoms ${ }^{14}$ and MPD of molecules. ${ }^{15}$ In these nonperturbative methods, the MPI or MPD rates are determined from the first principle through the use of the complex coordinate transformation $^{16,17}$ and $L^{2}$ (square-integrable) discretization ${ }^{18}$ of atomic and molecular continua. Extension to nonHermitian MMFT for polychromatic field problems is in progress and will be discussed elsewhere. ${ }^{19}$ In most problems in quantum optics, however, irreversible loss mechanisms in general are difficult to implement in a selfconsistent manner and one is usually led to the use of some sort of phenomenological damping constants. ${ }^{20}$ It is this second route we shall adopt in this paper.

In Sec. II, we shall first develop a general MMFT formalism for the problem of dissipative $N$-level systems interacting with several linearly polarized monochromatic classical fields. We then point out the general conditions in which coherent population trapping may take place. In Sec. III, we extend the generalized Van Vleck (GVV) nearly degenerate perturbation theory ${ }^{21,22}$ to an analytical treatment of the three-level trapping problem, incorporating the corrections due to non-RWA terms. In Sec. IV, we present the new equations of motion for the time evolution of the damping three-level coherent vector $\mathbf{S}(t)$, based on the group-SU(3) generators. As the three-level system evolves in response to external fields, its dynamic behavior is mirrored in the geometric motions of $\mathbf{S}(t)$. It is found that the initially eight-dimensional coherent vector $\mathbf{S}(t)$ evolves to a one-dimensional scalar at exact coherent population trapping condition. Detailed results and pictorial representations of the trapping and SU(3) dynamical evolution will be presented in Sec. IV. Atomic units will be used throughout unless otherwise specified.

\section{SEMICLASSICAL MANY-MODE FLOQUET THEORY FOR DISSIPATIVE $\boldsymbol{N}$-LEVEL SYSTEMS}

The time evolution of an arbitrary $N$-level system, with irreversible dissipative couplings with its surroundings, interacting with two intense linearly polarized monochromatic fields is governed, within the electric dipole approximation, by the time-dependent Schrödinger equation

$$
i \frac{d}{d t} \Psi(t)=[\hat{H}(t)-i \hat{G}] \Psi(t)
$$

In Eq. (1), the Hermitian Hamiltonian $\hat{H}(t)$ is defined as

$$
\hat{H}(t)=\hat{H}_{0}+V(t),
$$

where $\hat{H}_{0}$ is the unperturbed Hamiltonian of the $N$-level system with eigenenergies $\left\{E_{\alpha}\right\}$ and eigenfunctions $\left\{\psi_{\alpha}\right\}$, i.e.,

$$
\widehat{H}_{0} \psi_{\alpha}=E_{\alpha} \psi_{\alpha}(\alpha=1,2, \ldots, N),
$$

and $\widehat{V}(t)$ is the electric dipole interaction,

$$
\widehat{V}(t)=-\sum_{i=1}^{2} \boldsymbol{\mu} \cdot \mathbf{E}_{i}^{(0)} \cos \left(\omega_{i} t+\phi_{i}\right)
$$

with $\boldsymbol{\mu}$ the electric dipole moment operator, and $E_{i}^{(0)}, \omega_{i}$, and $\phi_{i}$ specifying, respectively, the amplitude, frequency, and initial phase of the $i$ th classical field. The phenomenological damping operator $\widehat{G}$ in Eq.(1) is introduced to take into account irreversible transitions away from the $N$-level system that are not explicitly considered. In the unperturbed $\left\{\psi_{\alpha}\right\}$ basis, $\widehat{G}$ is defined as

$$
\widehat{G} \psi_{\alpha}=g_{\alpha} \psi_{\alpha} \quad(\alpha=1,2, \ldots, N),
$$

where $g_{\alpha}$ are scalar damping constants. In this section we shall extend the Hermitian MMFT previously developed $^{2-4}(\hat{G}=\hat{0})$ to the present non-Hermitian case allowing for nonvanishing $\widehat{G}$.

The complex solutions of Eq. (1) can be formally written, in column vectors,

$$
\mathbf{Y}_{\beta}(t)=e^{-i q_{\beta} t} \mathbf{y}_{\beta}(t) \quad(\beta=1,2, \ldots, N),
$$

with components $y_{\alpha \beta}(t) \equiv\left\langle\psi_{\alpha} \mid \mathbf{y}_{\beta}(t)\right\rangle$ expressible in terms of a double Fourier series, namely,

$$
y_{\alpha \beta}(t)=\sum_{m_{1}=-\infty}^{\infty} \sum_{m_{2}=-\infty}^{\infty}\left\langle\alpha m_{1} m_{2} \mid q_{\beta}\right\rangle e^{i\left(m_{1} \omega_{1}+m_{2} \omega_{2}\right) t} .
$$

Here the expansion coefficients $\left\langle\alpha m_{1} m_{2} \mid q_{\beta}\right\rangle$ are components of the complex quasienergy state (QES) $\left|q_{\beta}\right\rangle$ expressed in terms of the generalized Floquet basis state $\left|\alpha m_{1} m_{2}\right\rangle=|\alpha\rangle \otimes\left|m_{1}\right\rangle \otimes\left|m_{2}\right\rangle$, where $\alpha$ is the system index $(\alpha=1,2, \ldots, N)$ and $m_{1}$ and $m_{2}$ arbitrary integers. The complex QES $\left|q_{\beta}\right\rangle$ are eigenfunctions of $\widehat{A}_{F}$, a nonHermitian, time-independent two-mode Floquet Hamiltonian, with quasienergy eigenvalues $q_{\beta}$ :

$$
\widehat{A}_{F}\left|q_{\beta}\right\rangle=q_{\beta}\left|q_{\beta}\right\rangle \text {. }
$$

In the $\left\{\left|\alpha m_{1} m_{2}\right\rangle\right\}$ basis, $\hat{A}_{F}$ is defined as

$$
\begin{aligned}
\left\langle\alpha m_{1} m_{2}\left|\hat{A}_{F}\right| \beta n_{1} n_{2}\right\rangle= & \left\langle\alpha m_{1} m_{2}\left|\hat{H}_{F}\right| \beta n_{1} n_{2}\right\rangle \\
& -i g_{\beta} \delta_{\alpha \beta} \delta_{m_{1} n_{1}} \delta_{m_{2} n_{2}},
\end{aligned}
$$

where $g_{\beta}$ 's are the phenomenological damping constants defined in Eq. (5), and $\hat{H}_{F}$ is simply the corresponding Hermitian two-mode Floquet Hamiltonian for the nondamping case: 


$$
\begin{aligned}
\left\langle\alpha m_{1} m_{2}\left|\hat{H}_{F}\right| \beta n_{1} n_{2}\right\rangle= & \mathscr{H}_{\alpha \beta}^{\left[m_{1}-n_{1}, m_{2}-n_{2}\right]} \\
& +\left(m_{1} \omega_{1}+m_{2} \omega_{2}\right) \delta_{\alpha \beta} \delta_{m_{1} n_{1}} \delta_{m_{2} n_{2}},
\end{aligned}
$$

and

$$
\begin{aligned}
\mathscr{H}_{\alpha \beta}^{\left[m_{1}, m_{2}\right]}= & E_{\alpha} \delta_{\alpha \beta} \delta_{m_{1}, 0} \delta_{m_{2}, 0} \\
-\frac{1}{2} \sum_{i=1}^{2}\left\langle\alpha\left|\mu \cdot \mathbf{E}_{i}^{(0)}\right| \beta\right\rangle & \left\langle e^{i m_{i} \phi_{i}}\left(\delta_{m_{i}, 1}+\delta_{m_{i},-1}\right) \delta_{m 0},\right.
\end{aligned}
$$

with

$$
\delta_{m 0}= \begin{cases}\delta_{m_{2}, 0} & \text { if } i=1 \\ \delta_{m_{1}, 0} & \text { if } i=2 .\end{cases}
$$

Some general properties of the non-Hermitian Hamiltonian $\widehat{A}_{F}$ are summarized below. The eigenvalue equation (8) can be written more generally as

$$
\hat{A}_{F}^{+}\left|\lambda_{\beta n_{1} n_{2}}\right\rangle=\lambda_{\beta n_{1} n_{2}}\left|\lambda_{\beta n_{1} n_{2}}\right\rangle
$$

with the three indices $\beta, n_{1}, n_{2}$ correlated with the three indices in the generalized Floquet basis set. The concomitant adjoint Hamiltonian $\hat{A}_{F}^{+}$satisfies

$$
\hat{A}_{F}^{+}\left|\epsilon_{\beta n_{1} n_{2}}\right\rangle=\epsilon_{\beta n_{1} n_{2}}\left|\epsilon_{\beta n_{1} n_{2}}\right\rangle
$$

in such a way that the eigenvalues are related by

$$
\epsilon_{\beta n_{1} n_{2}}=\lambda_{\beta n_{1} n_{2}}^{*}
$$

and the eigenfunctions by the biorthogonality relationship

$$
\left\langle\epsilon_{\beta n_{1} n_{2}} \mid \lambda_{\alpha m_{1} m_{2}}\right\rangle=\delta_{\beta \alpha} \delta_{n_{1} m_{1}} \delta_{n_{2} m_{2}}
$$

and the closure relationship

$$
\sum_{\beta, n_{1}, n_{2}}\left|\lambda_{\beta n_{1} n_{2}}\right\rangle\left\langle\epsilon_{\beta n_{1} n_{2}}\right|=\hat{1},
$$

for all indices $\left(\beta, n_{1}, n_{2}\right)$ and $\left(\alpha, m_{1}, m_{2}\right)$. In addition to the properties described above, the complex eigenvalues and eigenfunctions of $\hat{A}_{F}$ and $\hat{A}_{F}^{+}$also satisfy the following periodic relations:

$$
\begin{aligned}
& \lambda_{\alpha m_{1} m_{2}}=\lambda_{\alpha 00}+m_{1} \omega_{1}+m_{2} \omega_{2} \\
& \epsilon_{\alpha m_{1} m_{2}}=\epsilon_{\alpha 00}+m_{1} \omega_{1}+m_{2} \omega_{2} \\
& \left\langle\beta, n_{1}+k_{1}, n_{2}+k_{2} \mid \lambda_{\alpha, m_{1}+k_{1}, m_{2}+k_{2}}\right\rangle=\left\langle\beta n_{1} n_{2} \mid \lambda_{\alpha m_{1} m_{2}}\right\rangle
\end{aligned}
$$

and

$$
\left\langle\beta, n_{1}+k_{1}, n_{2}+k_{2} \mid \epsilon_{\alpha, m_{1}+k_{1}, m_{2}+k_{2}}\right\rangle=\left\langle\beta n_{1} n_{2} \mid \epsilon_{\alpha m_{1} m_{2}}\right\rangle .
$$

Moreover, if $\hat{A}_{F}^{T}=\mathbf{A}_{F}$, i.e., a symmetric matrix [this is the case when the phases $\phi_{1}=\phi_{2}=0$ in Eq. (10)], one has in addition

$$
\left\langle\beta n_{1} n_{2} \mid \lambda_{\alpha m_{1} m_{2}}\right\rangle=\left\langle\epsilon_{\alpha m_{1} m_{2}} \mid \beta n_{1} n_{2}\right\rangle \equiv\left\langle\beta n_{1} n_{2} \mid \epsilon_{\alpha m_{1} m_{2}}\right\rangle^{*} \text {. }
$$

Finally, the complex quasienergy eigenvalues of $\hat{A}_{F}$ satisfy the sum rules

$$
\sum_{\alpha=1}^{N} \operatorname{Re}\left(\lambda_{\alpha m_{1} m_{2}}\right)=\sum_{\alpha=1}^{N} E_{\alpha}+m_{1} \omega_{1}+m_{2} \omega_{2}
$$

and

$$
\sum_{\alpha=1}^{N} \operatorname{Im}\left(\lambda_{\alpha m_{1} m_{2}}\right)=-\sum_{\alpha=1}^{N} g_{\alpha},
$$

irrespective of the values of the double Fourier indices $m_{1}$ and $m_{2}$. Similar relations exist for the $\hat{A}_{F}^{+}$eigenvalues.

Formally the solution $\Psi(t)$, in a column vector, of Eq. (1) can be written as

$$
\Psi(t)=\overleftrightarrow{\mathrm{Y}}(t) \overleftrightarrow{\mathrm{Y}}-1\left(t_{0}\right) \Psi\left(t_{0}\right)
$$

where $t_{0}$ is the initial time. Here the $N \times N$ matrix $\overleftrightarrow{\mathrm{Y}}(t)$ is formed by the $N$ independent column vectors of Eq. (6), and $\overleftrightarrow{\mathrm{Y}}^{-1}(t)$ is the inverse of $\overleftrightarrow{\mathrm{Y}}(t)$. It can be readily shown that the row vectors $\left[\overleftrightarrow{\mathrm{Y}}^{-1}(t)\right]_{\beta}$ of the matrix $\overleftrightarrow{\mathrm{Y}}^{-1}(t)$ can be Fourier decomposed into

$$
\left[\overleftrightarrow{\mathrm{Y}}^{-1}(t)\right]_{\beta}=e^{i p_{\beta}^{*} t}\left[\overleftrightarrow{\mathrm{y}}^{-1}(t)\right]_{\beta}, \quad \beta=1,2, \ldots, N
$$

where

$$
\begin{aligned}
{\left[\overleftrightarrow{\mathrm{y}}^{-1}(t)\right]_{\beta \alpha} } & \equiv\left\langle y_{\beta}^{-1}(t) \mid \psi_{\alpha}\right\rangle \\
& =\sum_{m_{1}, m_{2}}\left\langle p_{\beta} \mid \alpha m_{1} m_{2}\right\rangle e^{-i\left(m_{1} \omega_{1}+m_{2} \omega_{2}\right) t}
\end{aligned}
$$

with $p_{\beta}$ 's and $\left|p_{\beta}\right\rangle$ 's being the eigenvalues and eigenvectors of the concomitant adjoint Hamiltonian $\mathbf{A}_{F}^{+}$. From Eqs. (8), (11), (12), (16), and (21), we can see that $q_{\beta}$ and $p_{\beta}$ are only defined modulo $\omega_{1}$ and modulo $\omega_{2}$. We can thus choose $q_{\beta}$ and $p_{\beta}$ such that

$$
q_{\beta}=\lambda_{\beta 00}
$$

and

$$
p_{\beta}=\epsilon_{\beta 00},
$$

and hence $p_{\beta}^{*}=q_{\beta}$. We note that Eqs. (16) and (21) have nothing to do with how we choose $q_{\beta}$ and $p_{\beta}$ from $\lambda_{\beta m_{1} m_{2}}$ 's and $\epsilon_{\beta m_{1} m_{2}}$ 's.

Analogous to the conventional practice for the nondamping case, we can also define a nonunitary time evolution operator for the damping case as

$$
\widehat{U}\left(t ; t_{0}\right) \equiv \overleftrightarrow{\mathrm{Y}}(t) \overleftrightarrow{\mathrm{Y}}-1\left(t_{0}\right)
$$

The expression of $\hat{U}\left(t ; t_{0}\right)$ in terms of the complex quasienergy eigenvalues and eigenvectors of $\hat{A}_{F}$ and $\hat{A}_{F}^{+}$ can be written as 


$$
\begin{array}{r}
\hat{U}_{\beta \alpha}\left(t ; t_{0}\right)=\sum_{\gamma} \sum_{m_{1}, m_{2}} \sum_{n_{1}, n_{2}}\left\langle\beta n_{1} n_{2} \mid \lambda_{\gamma 00}\right\rangle\left\langle\epsilon_{\gamma 00} \mid \alpha m_{1} m_{2}\right\rangle \\
\times \exp \left[i\left(n_{1} \omega_{1}+n_{2} \omega_{2}\right) t\right. \\
\left.-i\left(m_{1} \omega_{1}+m_{2} \omega_{2}\right) t_{0}\right] \\
\times \exp \left[-i \lambda_{\gamma 00}\left(t-t_{0}\right)\right],
\end{array}
$$

or more elegantly,

$$
\begin{aligned}
\hat{U}_{\beta \alpha}\left(t ; t_{0}\right) & \\
= & \sum_{n_{1}, n_{2}}\left\langle\beta n_{1} n_{2}\left|e^{-i \hat{A}_{F}\left(t-t_{0}\right)}\right| \alpha 00\right\rangle e^{i\left(n_{1} \omega_{1}+n_{2} \omega_{2}\right) t} .
\end{aligned}
$$

The transition probability for finding the system in the state $|\beta\rangle \equiv \psi_{\beta}$ at the instant of time $t$ when the system is initially, at time $t_{0}$, in the state $|\alpha\rangle \equiv \psi_{\alpha}$ is then

$$
\begin{aligned}
P_{\alpha \rightarrow \beta}^{\prime}\left(t ; t_{0}\right)=\left|\hat{U}_{\beta \alpha}\left(t ; t_{0}\right)\right|^{2} & \\
=\sum_{m_{1}, m_{2}} \sum_{k_{1}, k_{2}} & \left\langle\beta k_{1} k_{2}\left|e^{-i \hat{A}_{F}\left(t-t_{0}\right)}\right| \alpha 00\right\rangle \\
& \times \exp \left[+i\left(m_{1} \omega_{1}+m_{2} \omega_{2}\right) t_{0}\right] \\
& \times\left\langle\alpha m_{1} m_{2}\left|e^{i \hat{A}_{F}^{+}\left(t-t_{0}\right)}\right| \beta k_{1} k_{2}\right\rangle .
\end{aligned}
$$

Averaging over initial times $t_{0}$ while fixing the elapsed time $t-t_{0}$ in Eq. (27) results in

$$
P_{\alpha \rightarrow \beta}\left(t-t_{0}\right)=\sum_{k_{1}, k_{2}}\left|\left\langle\beta k_{1} k_{2}\left|e^{-i \hat{A}_{F}\left(t-t_{0}\right)}\right| \alpha 00\right\rangle\right|^{2}
$$

Here we have taken into account the fact that the initial time $t_{0}$, or equivalently the initial phase of the radiation fields encountered by the system, may be only randomly sampled in actual experiments. We remark that in deriving Eqs. (27)-(29), use has been made of the periodic relations, Eqs. (16), biorthogonality, Eq. (14), and the closure relation, Eq. (15).

Let us now consider the conditions under which coherent population trapping may occur. It is useful to first examine the long time average of the population distribution over various levels. Averaging over $t$, assuming $t_{0}=0$ in Eq. (28) yields

$$
\begin{aligned}
\bar{P}_{\alpha \rightarrow \beta}^{\prime} \equiv \lim _{T \rightarrow \infty}\left(\frac{1}{T} \int_{0}^{T} P_{\alpha \rightarrow \beta}^{\prime}(t)\right] \\
=\sum_{k_{1}, k_{2}} \sum_{m_{1}, m_{2}} \sum_{\gamma, l_{1}, l_{2}}\left\langle\beta k_{1} k_{2} \mid \lambda_{\gamma l_{1} l_{2}}\right\rangle\left\langle\epsilon_{\gamma l_{1} l_{2}} \mid \alpha 00\right\rangle\left\langle\alpha m_{1} m_{2} \mid \epsilon_{\gamma l_{1} l_{2}}\right\rangle\left\langle\lambda_{\gamma l_{1} l_{2}} \mid \beta k_{1} k_{2}\right\rangle \\
\quad \times \lim _{T \rightarrow \infty}\left\{\exp \left[2 \operatorname{Im}\left(\lambda_{\gamma l_{1} l_{2}}\right) T\right] /\left[2 \operatorname{Im}\left(\lambda_{\gamma l_{1} l_{2}}\right) T\right]\right\}
\end{aligned}
$$

while averaging over $\left(t-t_{0}\right)$ in Eq. (29) results in

$$
\bar{P}=\sum_{k_{1}, k_{2}} \sum_{\gamma, l_{1}, l_{2}}\left|\left\langle\beta k_{1} k_{2} \mid \lambda_{\gamma l_{1} l_{2}}\right\rangle\left\langle\epsilon_{\gamma l_{1} l_{2}} \mid \alpha 00\right\rangle\right|^{2} \lim _{T \rightarrow \infty}\left\{\exp \left[2 \operatorname{Im}\left(\lambda_{\gamma l_{1} l_{2}}\right) T\right] /\left[2 \operatorname{Im}\left(\lambda_{\gamma l_{1} l_{2}}\right) T\right]\right\} .
$$

It is easy to speculate, from Eqs. (26)-(31), that if the damping system is to survive the irreversible losses after an infinite time there must exist at least one real quasienergy eigenvalue $\lambda_{\mu l_{1} l_{2}}$, i.e., $\operatorname{Im}\left(\lambda_{\mu l_{1} l_{2}}\right)=0$, of the nonHermitian Floquet Hamiltonian $\hat{A}_{F}$. Here we note that (i) all imaginary parts, if not zero, of quasienergies are negative, this can be attributed to the non-negative diagonal matrix elements of $\widehat{G}$ of Eq. (1); and (ii) the imaginary part of each quasienergy is independent of the double Fourier indices, cf. Eq. (16). It is thus sufficient to consider only the behavior of $\operatorname{Im}\left(\lambda_{\mu 00}\right), \mu=1,2, \ldots, N$. The existence of at least one real quasienergy $\lambda_{\mu 00}$ for $\hat{A}_{F}$ possesses important meaning. Assuming only a few diagonal matrix elements of the $\widehat{G}$ operator, most likely associated with excited levels, are nonvanishing, it can be shown that ${ }^{23}$

$\operatorname{Im}\left(\lambda_{\mu 00}\right)=-|c|^{2} \sum_{\eta}^{\prime} g_{\eta} \sum_{k_{1}, k_{2}}\left|\left\langle\eta k_{1} k_{2} \mid \lambda_{\mu 00}\right\rangle\right|^{2} \leq 0$,

where $c$ is the proportionality constant such that $|c|^{2}\left\langle\lambda_{\mu 00} \mid \lambda_{\mu 00}\right\rangle=1$. Note that the prime appearing on the summation in Eq. (32) indicates only those unstable unperturbed states $|\eta\rangle$ characterized by damping constants $g_{\eta}>0$ need be summed over. Equation (32) implies that $\operatorname{Im}\left(\lambda_{\mu 00}\right)=0$, if and only if

$$
\left\langle\eta k_{1} k_{2} \mid \lambda_{\mu 00}^{\cdot}\right\rangle=\left\langle\eta k_{1} k_{2} \mid \epsilon_{\mu 00}\right\rangle=0 \text {. }
$$

Consequently, the quasienergy state $\left|\lambda_{\mu 00}\right\rangle$ with real eigenvalue is a coherent superposition of unperturbed 
stable levels $|\alpha\rangle$, i.e., those with $g_{\alpha}=0$. It is this coherent superposition state that will survive the irreversible dissipative external perturbations. We therefore conclude that the system can be trapped only if there exists some real quasienergies $\lambda_{\mu 00}$. As will be shown, however, in Sec. III that in practice it is difficult to prepare a superposition state with vanishing width when non-RWA factors become significant. Nevertheless, it is still experimentally feasible to observe the "quasitrapping" phenomenon. That is, the width of the superposition state, though not identically zero, is sufficiently small so that the system can be trapped for a sufficiently long period of time before it completely decays away.

\section{POPULATION TRAPPING IN DISSIPATIVE THREE-LEVEL SYSTEMS}

The general theory outlines in Sec. II enables us to explore the dynamical evolution of dissipative systems in the presence of polychromatic fields much beyond the limit of the often-used RWA. In order to study the salient feature of coherent population trapping under the influence of non-RWA terms, we shall focus in this section only on the most often studied cascade [Fig. 1(a)] or $\Lambda$ [Fig. 1(b)] type three-level systems. We shall assume the following. (i) The three levels are indexed by 1,2 , and 3 with the level 2 having opposite parity to the levels 1 and 3 . Thus under the electric dipole approximation, one-photon resonance transitions can occur only between levels 1 and 2, or between 2 and 3 . (ii) The three levels are sufficiently unequally spaced so that when illuminated by two intense, nearly resonant, monochromatic radiation fields, each resonant transition is predominantly driven by one field. (iii) Irreversible loss can only occur via the level 2 , the common level, with a loss rate $g_{2}$, whereas levels 1 and 3 are stable.

For the three-level systems described above in the presence of two strong linearly polarized and monochromatic radiation fields, the most important process is the one that one photon, say of angular frequency $\omega_{1}$, is resonantly absorbed (or emitted) between levels 1 and 2 , while the other photon, of different angular frequency $\omega_{2}$, is resonantly absorbed (or emitted) between levels 2 and 3, i.e., the twophoton resonance process. In addition to the two-photon resonance process, we may also expect that the multiphoton resonant processes (i.e., of more than two photons) can take place if the field strengths are large enough. In the following we shall consider cases where $\left|E_{2}-E_{1}\right| \approx\left(2 n_{1}+1\right) \omega_{1}$ and $\left|E_{3}-E_{2}\right| \approx\left(2 n_{2}+1\right) \omega_{2}$, with $n_{1}$ and $n_{2}=0,1,2, \ldots$. Under these two resonant

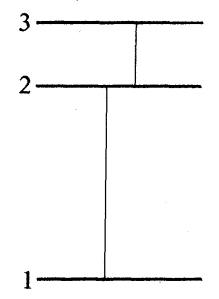

(a)

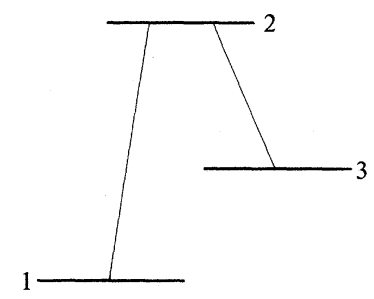

(b)
FIG. 1. (a) Cascade-type and (b) $\Lambda$-type three-level systems. conditions we expect that the transition is dominated by sequential absorptions (or emissions) of $\left(2 n_{1}+1\right)$ photons of frequency $\omega_{1}$ between levels 1 and 2 , and sequential absorptions (or emissions) of $\left(2 n_{2}+1\right)$ photons of $\omega_{2}$ between levels 2 and 3 . For convenience, we at first introduce notations

$$
\begin{aligned}
& b_{i}^{(\alpha \beta)}=-\frac{1}{2}\left\langle\alpha\left|\boldsymbol{\mu} \cdot \mathbf{E}_{i}^{(0)}\right| \beta\right\rangle e^{i \phi_{i}} \quad(i=1,2), \\
& \Delta_{n_{1}}=\left(E_{2}-E_{1}\right)-\left(2 n_{1}+1\right) \omega_{1},
\end{aligned}
$$

and

$$
\Delta_{n_{2}}^{\prime}= \begin{cases}\left(E_{3}-E_{2}\right)-\left(2 n_{2}+1\right) \omega_{2} & \text { for cascade type } \\ \left(E_{3}-E_{2}\right)+\left(2 n_{2}+1\right) \omega_{2} & \text { for } \Lambda \text { type }\end{cases}
$$

where $E_{\alpha}$ 's and $|\alpha\rangle$ 's are defined in Eq. (3), and $\mathbf{E}_{i}^{(0)}, \omega_{i}$, and $\phi_{i}$ are, respectively, the amplitude, angular frequency, and the initial phase of the $i$ th field. In order to apply perturbation methods to study the resonant processes experienced by the system, we shall further assume that $\left|b_{1}^{(12)}\right|,\left|b_{1}^{(23)}\right| \ll\left|E_{2}-E_{1}\right|$ and $\left|b_{2}^{(12)}\right|,\left|b_{2}^{(23)}\right|$ $\ll\left|E_{3}-E_{2}\right|$. The loss rate $g_{2}$ of the common level 2 is of course much smaller than $\left|E_{2}-E_{1}\right|$ and $\left|E_{3}-E_{2}\right|$. At the resonance conditions assumed above, we can easily find that diagonal matrix elements of the Hamiltonian $\hat{A}_{F}$ of Eq. (8),

$$
\begin{aligned}
& \left\langle 1,0,0\left|\hat{A}_{F}\right| 1,0,0\right\rangle, \\
& \left\langle 2,-\left(2 n_{1}+1\right), 0\left|\hat{A}_{F}\right| 2,-\left(2 n_{1}+1\right), 0\right\rangle,
\end{aligned}
$$

and

$$
\left\langle 3,-\left(2 n_{1}+1\right),-\left(2 n_{2}+1\right)\left|\hat{A}_{F}\right| 3,-\left(2 n_{1}+1\right),-\left(2 n_{2}+1\right)\right\rangle
$$

for the cascade type, or

$$
\left\langle 3,-\left(2 n_{1}+1\right),\left(2 n_{2}+1\right)\left|\hat{A}_{F}\right| 3,-\left(2 n_{1}+1\right),\left(2 n_{2}+1\right)\right\rangle
$$

for the $\Lambda$ type, are nearly degenerate. To exploit the analytical properties of the resonance trapping problem, it is most expedient to extend the generalized Van Vleck (GVV) nearly degenerate perturbation theory ${ }^{21,22}$ to the two-mode Floquet Hamiltonian $\hat{A}_{F}$. This method has been employed in our previous study of the SU(3) dynamical evolution of dissipationless three-level systems. ${ }^{4}$ It can be easily shown that the conventional (Hermitian) GVV approach $^{4,21,22}$ is extendable to a non-Hermitian Hamiltonian such as $\hat{A}_{F}$ by the use of the biorthogonal variational principle. ${ }^{17}$ The power of the GVV approach lies in the fact that it reduces the infinite-dimensional nonHermitian Floquet Hamiltonian $\hat{A}_{F}$ to a $3 \times 3$ effective non-Hermitian Hamiltonian $\hat{A}_{3}$ spanned by the three nearly degenerate unperturbed model space bases, $|100\rangle$, $\left|2,-\left(2 n_{1}+1\right), 0\right\rangle$, and $\left|3,-\left(2 n_{1}+1\right), \mp\left(2 n_{2}+1\right)\right\rangle$. In addition, if the perturbed model space wave functions are exact to the $n$th order, the corresponding Hamiltonian matrix on the model space can be computed up to the $(2 n+1)$ th order. ${ }^{21,22}$ Thus to the first order in the wave function, the model space Hamiltonian matrix is accurate up to the third order and can be expressed analytically as

$$
\widehat{A}_{3}=\widehat{A}_{3}^{(0)}+A_{3}^{(1)}+A_{3}^{(2)}+A_{3}^{(3)} \text {. }
$$


Assuming $E_{1}=0$, the $3 \times 3$ non-Hermitian matrix $\widehat{A}_{3}$ in Eq. (37) has the general form

$$
\hat{A}_{3}=\left[\begin{array}{ccc}
\widetilde{\delta} & \alpha_{n_{1}} & u \\
\alpha_{n_{1}}^{\prime} & \Delta_{n_{1}}+\widetilde{\delta}_{2}-i g_{2} & \beta_{n_{2}} \\
u^{\prime} & \beta_{n_{2}}^{\prime} & \Delta_{n_{1}}+\Delta_{n_{2}}^{\prime}+\widetilde{\delta}_{3}
\end{array}\right]
$$

where $\widetilde{\delta}_{1}, \widetilde{\delta}_{2}, \widetilde{\delta}_{3}, u, u^{\prime}$, and portions or all of $\alpha_{n_{1}}, \alpha_{n_{1}}^{\prime}, \beta_{n_{2}}$, and $\beta_{n_{2}}^{\prime}$ are perturbation corrections due to the external space of $\widetilde{A}_{F}$, i.e., the remainder of the configuration space other than the model space. Using Eq. (37), we thus have reduced Eq. (8) to a three-dimensional eigenvalue problem, namely,

$$
\widehat{A}_{3}\left|q_{\beta}\right\rangle=q_{\beta}\left|q_{\beta}\right\rangle \text {, }
$$

where the $q_{\beta}$ 's and $\left|q_{\beta}\right\rangle$ are the approximate quasienergy eigenvalues and eigenvectors correlated with the three nearly degenerate unperturbed Floquet bases $|100\rangle$, $\left|2,-\left(2 n_{1}+1\right), 0\right\rangle$, and $\left|3,-\left(2 n_{1}+1\right), \mp\left(2 n_{2}+1\right)\right\rangle$. In connection with Eq. (38), one finds that the study of the time development of the wave function, Eq. (1), can be greatly simplified in the rotating-wave frame (not the RWA) defined via the transformation

$$
\Psi_{R}(t)=\hat{R}(t) \Psi(t),
$$

where the unitary transformation matrix $\hat{R}(t)$ is given by

$$
\widehat{R}(t)=\left[\begin{array}{ccc}
1 & 0 & 0 \\
0 & e^{i\left(2 n_{1}+1\right) \omega_{1} t} & 0 \\
0 & 0 & e^{i\left[\left(2 n_{1}+1\right) \omega_{1} \pm\left(2 n_{2}+1\right) \omega_{2}\right] t}
\end{array}\right] .
$$

The time evolution operator, Eq. (25) or (27), is likewise simplified under the rotating-wave transformation $\hat{R}(t)$ :

$$
\widehat{U}_{R}\left(t ; t_{0}\right) \equiv \hat{R}(t) \hat{U}\left(t ; t_{0}\right) \cong e^{-i \hat{A}_{3}\left(t-t_{0}\right)},
$$

so that fast oscillating terms originally associated with $\widehat{U}\left(t ; t_{0}\right)$ are totally quenched. Combining Eqs. (39) and (42), one gets

$$
\left(\hat{U}_{R}\right)_{\beta \gamma}(t ; 0)=\sum_{\gamma=1}^{3} e^{-i q_{\gamma} t}\left\langle\beta \mid q_{\gamma}\right\rangle\left\langle p_{\gamma} \mid \gamma\right\rangle,
$$

where $\left|p_{\gamma}\right\rangle$ are eigenstates of the adjoint eigenvalue equation

$$
A_{3}^{+}\left|p_{\gamma}\right\rangle=p_{\gamma}\left|p_{\gamma}\right\rangle
$$

with $p_{\gamma}=q_{\gamma}^{*}$, and $|\alpha\rangle,|\beta\rangle, \ldots$ denoting unperturbed Floquet states $|100\rangle, \quad\left|2,-\left(2 n_{1}+1\right), 0\right\rangle$, or $\left|3,-\left(2 n_{1}+1\right), \mp\left(2 n_{2}+1\right)\right\rangle$. In the following we shall confine our discussions in the rotating frame defined in Eqs. (39)-(41), and drop the subscript $R$ in Eq. (42) unless necessary for clarification.

\section{A. Two-photon resonance trapping}

In this subsection we shall study the two-photon resonance trapping condition based on the GVV perturbation theory. The $3 \times 3$ effective non-Hermitian Hamiltonian
$\widehat{A}_{3}$ in Eqs. (37) and (38) can be written more explicitly as

$$
\widehat{A}_{3}=\left[\begin{array}{ccc}
\delta_{1}-i \delta_{1}^{\prime} & \alpha+\delta_{12} & \delta_{13}-i \delta_{13}^{\prime} \\
\alpha^{*}+\delta_{12}^{\prime} & \Delta+\delta_{2}-i\left(g_{2}+\delta_{2}^{\prime}\right) & \beta+\delta_{23} \\
\delta_{13}^{*}-i \delta_{13}^{\prime *} & \beta^{*}+\delta_{23}^{\prime} & \Delta+\Delta^{\prime}+\delta_{3}-i \delta_{3}^{\prime}
\end{array}\right]
$$

where $\alpha=b_{1}^{(12)}, \beta=b_{2}^{(23)}, \Delta=\Delta_{0}$, and $\Delta^{\prime}=\Delta_{0}^{\prime}$. In Eq. (44), $\alpha$ and $\beta$ are the GVV first-order corrections (due to $A_{3}^{(1)}$ ), $\left(\delta_{1}-i \delta_{1}^{\prime}\right),\left(\delta_{2}-i \delta_{2}^{\prime}\right),\left(\delta_{3}-i \delta_{3}^{\prime}\right)$, and $\left(\delta_{13}-i \delta_{13}^{\prime}\right)$ are the GVV second-order corrections (due to $\widehat{A}_{3}^{(2)}$ ), and $\delta_{12}, \delta_{12}^{\prime}$, $\delta_{23}$, and $\delta_{23}^{\prime}$ are the GVV third-order corrections (due to $\left.\hat{A}_{3}^{(3)}\right)$. In light of the smallness of the third-order corrections compared with other lower-order corrections, these third-order terms will be totally neglected in the following analysis. The second-order corrections can be expressed in simple analytical forms as follows:

$$
\begin{aligned}
& \delta_{1}=-\frac{1}{\omega_{21}+\omega_{1}}\left|b_{1}^{(12)}\right|^{2}-\frac{2 \omega_{21}}{\omega_{21}^{2}-\omega_{2}^{2}}\left|b_{2}^{(12)}\right|^{2}, \\
& \delta_{3}=-\frac{1}{\omega_{32} \pm \omega_{2}}\left|b_{2}^{(23)}\right|^{2}+\frac{2 \omega_{32}}{\omega_{32}^{2}-\omega_{1}^{2}}\left|b_{1}^{(23)}\right|^{2}, \\
& \delta_{2}=-\left(\delta_{1}+\delta_{3}\right), \\
& \delta_{13}=\frac{1}{2}\left[\frac{1}{\omega_{32}-\omega_{1}}+\frac{1}{-\omega_{21} \pm \omega_{2}} \mid b_{2}^{(12)} b_{1}^{(23)},\right. \\
& \delta_{1}^{\prime}=\left[\frac{1}{\left(\omega_{21}+\omega_{1}\right)^{2}}\left|b_{1}^{(12)}\right|^{2}+2 \frac{\omega_{21}^{2}+\omega_{2}^{2}}{\left(\omega_{21}^{2}-\omega_{2}^{2}\right)^{2}}\left|b_{2}^{(12)}\right|^{2}\right] g_{2}, \\
& \delta_{3}^{\prime}=\left[\frac{1}{\left(\omega_{32} \pm \omega_{2}\right)^{2}}\left|b_{2}^{(23)}\right|^{2}+2 \frac{\omega_{32}^{2}+\omega_{1}^{2}}{\left(\omega_{32}^{2}-\omega_{1}^{2}\right)^{2}}\left|b_{1}^{(23)}\right|^{2}\right] g_{2},
\end{aligned}
$$

$\delta_{2}^{\prime}=-\left(\delta_{1}^{\prime}+\delta_{3}^{\prime}\right)$,

and

$\delta_{13}^{\prime}=\frac{1}{2}\left[\frac{1}{\left(\omega_{32}-\omega_{1}\right)^{2}}+\frac{1}{\left(-\omega_{21} \pm \omega_{2}\right)^{2}}\right] b_{2}^{(12)} b_{1}^{(23)} g_{2}$,

where $\omega_{21}=\left|E_{2}-E_{1}\right|, \omega_{32}=\left|E_{3}-E_{2}\right|$, and when the \pm sign simultaneously appears, the positive sign is for the cascade-type, while the negative sign is for the $\Lambda$-type system. We see that the first terms of each of Eqs. (45a), (45b), (46a), and (46b) are simply the bichromatic BlochSiegert shifts caused by the antirotating effect in the twolevel case, ${ }^{3}$ whereas each of the corresponding second terms is the manifestation of the effect due to non-RWA transition channel. (We recall that by assuming $\omega_{21} \sim \omega_{1}$, $\omega_{32} \sim \omega_{2}$, and that $\omega_{21}$ and $\omega_{32}$ are widely different, the dominant transition is the RWA channel $|1\rangle \stackrel{\omega_{1}}{\rightarrow}|2\rangle \stackrel{\omega_{2}}{\rightarrow}|3\rangle$. The non-RWA channel is the secon- 
dary channel described by $|1\rangle \stackrel{\omega_{2}}{\rightarrow}|2\rangle \stackrel{\omega_{1}}{\rightarrow}|3\rangle$.)

In the conventional RWA, i.e., neglecting both the antirotating effect and the secondary transition channel, coherent trapping is expected when $\Delta+\Delta^{\prime}=0$, the exact two-photon resonance, and one of the three roots of the RWA secular equation is real (zero if $\left.E_{1}=0\right)^{8-10}$ By including the non-RWA corrections, the trapping conditions are modified and much more complicated.

We have considered two different ways of obtaining the modified trapping conditions. The first method is to search for the real root directly from the determinant $D(\lambda) \equiv \operatorname{det}\left|\hat{A}_{3}-\lambda \hat{\mathbb{1}}\right|=0$. Imposing the condition that $\lambda$ be real, one finds that the cubic polynomial for the real part of $D(\lambda)$.

$$
f(\lambda) \equiv-\lambda^{3}+\left(2 \Delta+\Delta^{\prime}\right) \lambda^{2}-B \lambda+C=0,
$$

and the quadratic equation for the imaginary part of $D(\lambda)$,

$$
h(\lambda) \equiv g_{2} \lambda^{2}-F \lambda+G=0
$$

must have common real solutions for $\lambda$. Here $B, C, F$, and $G$ are sophisticated functions of detunings $\Delta$ and $\Delta^{\prime}$ and Rabi frequencies $\alpha$ and $\beta$, and are given by

$$
\begin{aligned}
B= & \delta_{1}\left(2 \Delta+\Delta^{\prime}+\delta_{2}+\delta_{3}\right)-\delta_{1}\left(g_{2}+\delta_{2}^{\prime}+\delta_{3}^{\prime}\right)+\left(\Delta+\delta_{2}\right)\left(\Delta+\Delta^{\prime}+\delta_{3}\right)-\left(g_{2}+\delta_{2}^{\prime}\right) \delta_{3}^{\prime}-|\alpha|^{2}-|\beta|^{2}-\left(\left|\delta_{13}\right|^{2}-\left|\delta_{13}^{\prime}\right|^{2}\right), \\
C= & {\left[\delta_{1}\left(\Delta+\delta_{2}\right)-\delta_{1}^{\prime}\left(g_{2}+\delta_{2}^{\prime}\right)\right]\left(\Delta+\Delta^{\prime}+\delta_{3}\right)-\delta_{3}^{\prime}\left[\delta_{1}\left(g_{2}+\delta_{2}^{\prime}\right)+\delta_{1}^{\prime}\left(\Delta+\delta_{2}\right)\right] } \\
& \quad+2 \operatorname{Re}\left(\alpha \beta \delta_{13}^{*}\right)-|\beta|^{2} \delta_{1}-|\alpha|^{2}\left(\Delta+\Delta^{\prime}+\delta_{3}\right)-\left[\left(\left|\delta_{13}\right|^{2}-\left|\delta_{13}^{\prime}\right|^{2}\right)\left(\Delta+\delta_{2}\right)-2 \operatorname{Re}\left(\delta_{13} \delta_{13}^{\prime}\right)\left(g_{2}+\delta_{2}^{\prime}\right)\right], \\
F= & \delta_{1}\left(g_{2}+\delta_{2}^{\prime}+\delta_{3}^{\prime}\right)+\delta_{1}^{\prime}\left(2 \Delta+\Delta^{\prime}+\delta_{2}+\delta_{3}\right)+\left(\Delta+\delta_{2}\right) \delta_{3}^{\prime}+\left(g_{2}+\delta_{2}^{\prime}\right)\left(\Delta+\Delta^{\prime}+\delta_{3}\right)-2 \operatorname{Re}\left(\delta_{13} \delta_{13}^{\prime *}\right),
\end{aligned}
$$

and

$$
\begin{aligned}
G= & {\left[\delta_{1}\left(\Delta+\delta_{2}\right)-\delta_{1}^{\prime}\left(g_{2}+\delta_{2}^{\prime}\right)\right] \delta_{3}+\left[\delta_{1}\left(g_{2}+\delta_{2}^{\prime}\right)+\delta_{1}^{\prime}\left(\Delta+\delta_{2}\right)\right]\left(\Delta+\Delta^{\prime}+\delta_{3}\right) } \\
& +2 \operatorname{Re}\left(\alpha \beta \delta_{13}^{\prime *}\right)-|\beta|^{2} \delta_{1}^{\prime}-|\alpha|^{2} \delta_{3}^{\prime}-\left[\left(\left|\delta_{13}\right|^{2}-\left|\delta_{13}^{\prime}\right|^{2}\right)\left(g_{2}+\delta_{2}^{\prime}\right)+2 \operatorname{Re}\left(\delta_{13} \delta_{13}^{\prime *}\right)\left(\Delta+\delta_{2}\right)\right]
\end{aligned}
$$

Let the solutions of the quadratic equation $h(\lambda)=0$ be $\lambda_{ \pm}, \lambda_{ \pm}=\left[F \pm\left(F^{2}-4 g_{2} G\right)^{1 / 2}\right] / 2 g_{2}$.

If either of $\lambda_{+}$is also a solution of Eq. (47a), we must have $f\left(\lambda_{+}\right)=0$ or $f\left(\lambda_{-}\right)=0$. This leads to rather intractable analytical results. In particular, one finds that the trapping conditions obtained from $f\left(\lambda_{ \pm}\right)=0$ are rather difficult to meet precisely, if not possible.

To obtain better physical insights regarding the population trapping process near two-photon resonance, we resort to the following second method based on an ordinary perturbation treatment of $\hat{A}_{3}$. Writing

$$
\widehat{A}_{3}=\widehat{A}_{30}+\widehat{V}_{3},
$$

where $A_{30}=A_{3}^{(0)}+A_{3}^{(1)}$ is precisely the RWA Hamiltoni$\mathrm{an}^{4}$ at two-photon resonance,

$$
\widehat{A}_{30}=\left[\begin{array}{ccc}
0 & \alpha & 0 \\
\alpha^{*} & \Delta-i g_{2} & \beta \\
0 & \beta^{*} & 0
\end{array}\right]
$$

and the perturbation term $\widehat{V}_{3}=\widehat{A}_{3}^{(0)}$ (ignoring the small third-order correction term $\widehat{A}_{3}^{(3)}$ ) is given by

$$
\widehat{V}_{3}=\left[\begin{array}{ccc}
\delta_{1}-i \delta_{1}^{\prime} & 0 & \delta_{13}-i \delta_{13}^{\prime} \\
0 & \delta_{2}-i \delta_{2}^{\prime} & 0 \\
\delta_{13}^{*}-i\left(\delta_{13}^{\prime}\right)^{*} & 0 & \delta+\delta_{3}-i \delta_{3}
\end{array}\right]
$$

where $\delta=\Delta+\Delta^{\prime}$ is small, of the same order of magnitude as $\delta_{1}, \delta_{2}, \delta_{3}$, and $\delta_{13}$. Let the solutions of $\widehat{A}_{3}\left|\lambda_{i}\right\rangle=\lambda_{i}\left|\lambda_{i}\right\rangle, i=1,2,3$, be expanded in perturbation series,

$$
\left|\lambda_{i}\right\rangle=\left|\lambda_{i}^{(0)}\right\rangle+\left|\lambda_{i}^{(1)}\right\rangle+\left|\lambda_{i}^{(2)}\right\rangle+\cdots
$$

and

$$
\lambda_{i}=\lambda_{i}^{(0)}+\lambda_{i}^{(1)}+\lambda_{i}^{(2)}+\cdots .
$$

The zeroth-order terms $\lambda_{i}^{(0)}$ and $\left|\lambda_{i}^{(0)}\right\rangle$ are the solutions of the unperturbed (RWA) Hamiltonian $\hat{A}_{30}$, namely,

$$
\begin{aligned}
& \lambda_{1}^{(0)}=0, \\
& \left.\lambda_{2,3}^{(0)}=\frac{1}{2}\left(\Delta-i g_{2}\right) \pm \frac{1}{2}\left[\left(\Delta-i g_{2}\right)^{2}+4 \Omega^{2}\right)\right]^{1 / 2} \\
& \text { with } \Omega=\left(|\alpha|^{2}+|\beta|^{2}\right)^{1 / 2}, \\
& \left|\lambda_{1}^{(0)}\right\rangle=\frac{1}{\Omega}\left[\begin{array}{c}
-\beta \\
0 \\
\alpha^{*}
\end{array}\right], \quad\left|\lambda_{1}^{(0) *}\right\rangle=\frac{1}{\Omega}\left[\begin{array}{c}
-\beta \\
0 \\
\alpha^{*}
\end{array}\right], \\
& \left|\lambda_{2,3}^{(0)}\right\rangle=\frac{1}{\left(\Omega^{2}+\lambda_{2,3}^{(0) 2}\right)^{1 / 2}}\left[\begin{array}{c}
\alpha \\
\lambda_{2,3}^{(0)} \\
\beta^{*}
\end{array}\right], \\
& \left|\lambda_{2,3}^{(0) *}\right\rangle=\frac{1}{\left(\Omega^{2}+\lambda_{2,3}^{(0) * 2}\right)^{1 / 2}}\left[\begin{array}{c}
\alpha \\
\lambda_{2,3}^{(0) *} \\
\beta
\end{array}\right],
\end{aligned}
$$

and

$$
\left\langle\lambda_{i}^{(0) *} \mid \lambda_{j}^{(0)}\right\rangle=\delta_{i j} \quad \text { (biorthogonality). }
$$

Using a relation such as Eq. (32), one can readily show that

$$
\operatorname{Im}\left(\lambda_{2,3}^{(0)}\right)=-\left(\frac{\left|\lambda_{2,3}^{(0)}\right|^{2}}{\Omega^{2}+\left|\lambda_{2,3}^{(0)}\right|^{2}}\right) g_{2},
$$

and 


$$
\operatorname{Re}\left(\lambda_{2,3}^{(0)}\right)=\left(\frac{\left|\lambda_{2,3}^{(0)}\right|^{2}}{\left|\lambda_{2,3}^{(0)}\right|^{2}-\Omega^{2}}\right) \Delta
$$

Thus $\operatorname{Im}\left(\lambda_{2,3}^{(0)}\right)$ has the same order of magnitude as $g_{2}$, while $\operatorname{Re}\left(\lambda_{2,3}^{(0)}\right)$ is of the same order of magnitude as $|\alpha|$ or $|\beta|$. Carrying out the perturbation calculations to first order in the wave function, and second order in energies, we obtain $\operatorname{Im}\left(\lambda_{1}\right)$, the quantity of physical interest,

$$
\begin{aligned}
\operatorname{Im}\left(\lambda_{1}\right) & \simeq \operatorname{Im}\left(\lambda_{1}^{(0)}+\lambda_{1}^{(1)}+\lambda_{1}^{(2)}\right) \\
& =-\left(g_{2} / \Omega^{2}\right)\left[s_{1}+s_{2}\left(s_{2}^{\prime}-s_{2}^{\prime \prime}\right)\right],
\end{aligned}
$$

where

$$
\begin{aligned}
& s_{1}=|\beta|^{2} \delta_{1}^{\prime \prime}+|\alpha|^{2} \delta_{3}^{\prime \prime}-2 \operatorname{Re}\left(\alpha^{*} \beta^{*} \delta_{13}^{\prime \prime}\right), \\
& s_{2}=\left|\alpha^{*} \beta\left(-\delta_{1}+\delta+\delta_{3}\right)+\left(\alpha^{* 2} \delta_{13}-\beta^{2} \delta_{13}^{*}\right)\right|^{2}, \\
& s_{2}^{\prime}=\left[\left(\Omega^{2}+\left|\lambda_{2}^{(0)}\right|^{2}\right)\left|\Omega^{2}+\lambda_{2}^{(0) 2}\right|\right]^{-1}, \\
& s_{2}^{\prime \prime}=\left[\left(\Omega^{2}+\left|\lambda_{3}^{(0)}\right|^{2}\right)\left|\Omega^{2}+\lambda_{3}^{(0) 2}\right|\right]^{-1},
\end{aligned}
$$

with

$$
\begin{aligned}
& \delta_{1}^{\prime \prime}=\frac{|\alpha|^{2}}{\left(\omega_{21}+\omega_{1}\right)^{2}}+2 \frac{\left(\omega_{21}^{2}+\omega_{2}^{2}\right)}{\left(\omega_{21}^{2}-\omega_{2}^{2}\right)^{2}}\left|b_{2}^{(12)}\right|^{2}, \\
& \delta_{3}^{\prime \prime}=\frac{|\beta|^{2}}{\left(\omega_{32} \pm \omega_{2}\right)^{2}}+2 \frac{\left(\omega_{32}^{2}+\omega_{1}^{2}\right)}{\left(\omega_{32}^{2}-\omega_{1}^{2}\right)^{2}}\left|b_{1}^{(23)}\right|^{2},
\end{aligned}
$$

$$
\delta_{13}^{\prime \prime}=\frac{1}{2} b_{2}^{(12)} b_{1}^{(23)}\left(\frac{1}{\left(\omega_{32}-\omega_{1}\right)^{2}}+\frac{1}{\left(-\omega_{21} \pm \omega_{2}\right)^{2}}\right)
$$

Note that the first-order correction term $\operatorname{Im}\left(\lambda_{1}^{(1)}\right) \sim s_{1}$ and the second-order correction term $\operatorname{Im}\left(\lambda_{1}^{(2)}\right) \sim s_{2}\left(s_{2}^{\prime}+s_{2}^{\prime \prime}\right)$ are equally important, i.e., both are in the order of magnitude of $\left(\Omega^{2} / \omega^{2}\right) g_{2}$, where $\omega$ is either $\omega_{1}, \omega_{2}$, or $\left|\omega_{1}-\omega_{2}\right|$, and $\Omega^{2} / \omega^{2} \ll 1$. Equations (54) and (55) indicate that although the exact trapping condition, namely, $\operatorname{Im}\left(\lambda_{1}\right) \equiv 0$, would be difficult to match in practice, nevertheless one is still able to find a wide range of physical parameters such that $\operatorname{Im}\left(\lambda_{1}\right) / g_{2}<1$, so that the system can be trapped for a sufficiently long period of time, say $t_{\infty}$, with $t_{\infty} \gg 1 / g_{2}$. We shall refer this phenomenon as the quasitrapping.

Having clarified the physical situation, we can now write down several useful analytical expressions for the resonant two-photon quasitrapping process. Since $\operatorname{Im}\left(\lambda_{2,3}\right) \gg \operatorname{Im}\left(\lambda_{1}\right)$, only the physical states associated with the quasienergy $\lambda_{1}$ will be quasitrapped. The timeevolution operator at large times $t_{\infty} \gg 1 / g_{2}$ thus becomes

$$
U_{\beta \alpha}\left(t_{\infty} ; 0\right) \simeq e^{-2\left|\operatorname{Im}\left(\lambda_{1}\right)\right| t_{\infty}}\left\langle\beta \mid \lambda_{1}\right\rangle\left\langle\lambda_{1}^{*} \mid \alpha\right\rangle .
$$

Assuming the system is initially prepared in the ground level $|1\rangle$, one finds from Eq. (56) that the probabilities of having the system at large times $t_{\infty}$ in levels 1,2 , and 3 are, respectively,

$P_{1 \rightarrow 1}\left(t_{\infty}\right) \cong\left[\frac{|\beta|^{4}}{\Omega^{4}}-\frac{2 \Delta|\beta|^{2}}{\Omega^{8}}\left[2|\alpha|^{2}|\beta|^{2}\left(-\delta_{1}+\delta+\delta_{3}\right)+\left(|\alpha|^{2}-|\beta|^{2}\right)\left(\alpha \beta \delta_{13}^{*}+\right.\right.\right.$ c.c. $\left.\left.)\right]\right] e^{-2\left|\operatorname{Im}\left(\lambda_{1}\right)\right| t_{\infty}}$,

$P_{1 \rightarrow 2}\left(t_{\infty}\right) \cong 0$,

and

$$
\begin{aligned}
P_{1 \rightarrow 3}\left(t_{\infty}\right) \simeq\left[\begin{array}{r}
\frac{|\alpha|^{2}|\beta|^{2}}{\Omega^{4}}-\frac{1}{\Omega^{8}}\left\{\left[|\alpha|^{2}|\beta|^{2}\left(|\beta|^{2}-|\alpha|^{2}\right)\left(-\delta_{1}+\delta+\delta_{3}\right)\right.\right. \\
\left.\left.\left.+2|\alpha|^{2}|\beta|^{2} \alpha \beta \delta_{13}^{*}-\alpha^{*} \beta^{*}\left(|\alpha|^{4}+|\beta|^{4}\right) \delta_{13}\right]\left(\Delta+i g_{2}\right)+\text { c.c. }\right\}\right] e^{-2\left|\operatorname{Im}\left(\lambda_{1}\right)\right| t_{\infty}}
\end{array}\right.
\end{aligned}
$$

Thus the system is said to be quasitrapped in levels 1 and 3. Note the leading terms in Eqs. (57a) and (57c) are just the RWA expressions, namely,

$$
P_{1 \rightarrow 1}(\text { RWA })=|\beta|^{4} / \Omega^{4}
$$

and

$$
P_{1 \rightarrow 3}(\text { RWA })=|\alpha|^{2}|\beta|^{2} / \Omega^{4} .
$$

\section{B. Multiphoton resonance trapping}

For the cases $n_{1} \neq 0$ and/or $n_{2} \neq 0$, we expect that the resonant transition $|1\rangle \stackrel{\left(2 n_{1}+1\right) \omega_{1}}{\rightarrow}|2\rangle \stackrel{\left(2 n_{2}+1\right) \omega_{2}}{\rightarrow}|3\rangle$ shall dominate the rest of the $\left[\left(2 n_{1}+1\right)+\left(2 n_{2}+1\right)\right] ! /$ $\left(2 n_{1}+1\right) !\left(2 n_{2}+1\right)$ ! transition channels. By considering the three nearly degenerate Floquet states $|100\rangle$, $\left|2,-\left(2 n_{1}+1\right), 0\right\rangle$, and $\left|3,-\left(2 n_{1}+1\right), \mp\left(2 n_{2}+1\right)\right\rangle$ and employing the non-Hermitian GVV procedure, it is easy to find out that in Eq. (38), corrections $\widetilde{\delta}_{1}, \widetilde{\delta}_{2}$, and $\widetilde{\delta}_{3}$ are of second order, and $\left(\alpha_{n_{1}}, \alpha_{n_{1}}^{\prime}\right)$ and $\left(\beta_{n_{2}}, \beta_{n_{2}}^{\prime}\right)$ are, respectively, of $\left(2 n_{1}+1\right)$ th and $\left(2 n_{2}+1\right)$ th order, whereas $u$ and $u^{\prime}$ are of $\left[\left(2 n_{1}+1\right)+\left(2 n_{2}+1\right)\right]$ th order. Thus to an excellent approximation, $u$ and $u^{\prime}$ may be ignored, and one can replace the multiphoton $3 \times 3$ effective Floquet Hamiltonian, Eq. (38), by the following tridiagonal matrix [cf. Eq. (44)]:

$\hat{A}_{3}=\left[\begin{array}{ccc}\delta_{1}-i \delta_{1}^{\prime} & \alpha_{n_{1}} & 0 \\ \alpha_{n_{1}}^{*} & \Delta_{n_{1}}+\delta_{2}-i\left(g_{2}+\delta_{2}^{\prime}\right) & \beta_{n_{2}} \\ 0 & \beta_{n_{2}}^{*} & \Delta_{n_{1}}+\Delta_{n_{2}}^{\prime}+\delta_{3}-i \delta_{3}^{\prime}\end{array}\right]$, 
where the correction terms are given by

$$
\begin{aligned}
\delta_{1}= & -\left[\frac{1}{\left(2 n_{1}+2\right) \omega_{1}}+\vartheta\left(n_{1}\right) \frac{1}{2 n_{1} \omega_{1}}\right]\left(b_{1}^{(12)}\right)^{2} \\
& -\left(\frac{1}{\left(2 n_{1}+1\right) \omega_{1}+\omega_{2}}+\frac{1}{\left(2 n_{1}+1\right) \omega_{1}-\omega_{2}}\right)\left(b_{2}^{(12)}\right)^{2},
\end{aligned}
$$

(60a)

$$
\begin{aligned}
& \delta_{3}=\left[\left(\frac{1}{\omega_{1}+\left(2 n_{2}+1\right) \omega_{2}}+\frac{1}{-\omega_{1}+\left(2 n_{2}+1\right) \omega_{2}}\right]\left(b_{1}^{(23)}\right)^{2}\right. \\
& +\left(\frac{1}{\left(2 n_{2}+2\right) \omega_{2}}+\vartheta\left(n_{2}\right) \frac{1}{2 n_{2} \omega_{2}}\right]\left(b_{2}^{(23)^{2}}\right] \\
& \times\left\{\begin{array}{l}
1 \text { for cascade type } \\
-1 \text { for } \Lambda \text { type }
\end{array}\right.
\end{aligned}
$$

$$
\begin{aligned}
\delta_{2}= & -\left(\delta_{1}+\delta_{3}\right) \\
\delta_{1}^{\prime}= & {\left[\frac{1}{\left(2 n_{1}+2\right)^{2} \omega_{1}^{2}}+\vartheta\left(n_{1}\right) \frac{1}{4 n_{1}^{2} \omega_{1}^{2}}\right]\left(b_{1}^{(12)}\right)^{2} } \\
& +\left[\frac{1}{\left[\left(2 n_{1}+1\right) \omega_{1}+\omega_{2}\right]^{2}}\right. \\
\delta_{3}^{\prime}= & \left.\left.+\frac{1}{\left[\omega_{1}+\left(2 n_{2}+1\right) \omega_{2}\right]^{2}}\right]\left(b_{2}^{(12)}\right)^{2}\right] g_{2}, \\
& \left.+\frac{1}{\left[\left(2 n_{1}+1\right) \omega_{1}-\omega_{2}\right]^{2}}\right]\left(\frac{1}{\left[-\omega_{1}+\left(2 n_{2}+1\right) \omega_{2}\right]^{2}}\right]\left(b_{1}^{(23)}\right)^{2} \\
& \left.+\left[\frac{1}{\left(2 n_{2}+1\right)^{2} \omega_{2}^{2}+\vartheta\left(n_{2}\right) \frac{1}{4 n_{2}^{2} \omega_{2}^{2}}}\right]\left(b_{2}^{(23)}\right)^{2}\right] g_{2},
\end{aligned}
$$

$\delta_{2}^{\prime}=-\left(\delta_{1}^{\prime}+\delta_{2}^{\prime}\right)$,

$\alpha_{n_{1}}=\frac{(-1)^{n_{1}}\left(b_{1}^{(12)}\right)^{2 n+1}}{2^{2 n_{1}}\left(n_{1} !\right)^{2} \omega_{1}^{2 n_{1}}}$,

and

$$
\beta_{n_{2}}=\frac{(-1)^{n_{2}}\left(b_{2}^{(23)}\right)^{2 n_{2}+1}}{2^{2 n_{2}}\left(n_{2} !\right)^{2} \omega_{2}^{2 n_{2}}},
$$

with

$$
\vartheta(n)= \begin{cases}0 & \text { for } n=0 \\ 1 & \text { otherwise }\end{cases}
$$

By similar derivations made for the two-photon resonance case, i.e., Sec. III A, we can immediately conclude that the multiphoton resonance trapping will most likely occur when the condition

$$
\Delta_{n_{1}}+\Delta_{n_{2}}^{\prime}+\delta_{3}=\delta_{1}
$$

is satisfied. And the probabilities of finding the system in levels 1 and 3 at some large times $t_{\infty} \gg 1 / g_{2}$ are

$P_{1 \rightarrow 1}\left(t_{\infty}\right)=\frac{\left|\beta_{n_{2}}\right|^{4}}{\left(\left|\alpha_{n_{1}}\right|^{2}+\left|\beta_{n_{2}}\right|^{2}\right)^{2}} \exp \left[-2\left|\operatorname{Im}\left(q_{1}\right)\right| t_{\infty}\right]$,

and

$P_{1 \rightarrow 3}\left(t_{\infty}\right)=\frac{\left|\alpha_{n_{1}}\right|^{2}\left|\beta_{n_{2}}\right|^{2}}{\left(\left|\alpha_{n_{1}}\right|^{2}+\left|\beta_{n_{2}}\right|^{2}\right)^{2}} \exp \left[-2\left|\operatorname{Im}\left(q_{1}\right)\right| t_{\infty}\right]$,

where

$$
\left|\operatorname{Im}\left(q_{1}\right)\right|=\frac{\left|\beta_{n_{2}}\right|^{2} \delta_{1}^{\prime}+\left|\alpha_{n_{1}}\right|^{2} \delta_{3}^{\prime}}{\left(\left|\alpha_{n_{1}}\right|^{2}+\left|\beta_{n_{2}}\right|^{2}\right)} .
$$

with $\delta_{1}^{\prime}$ and $\delta_{3}^{\prime}$ defined in Eqs. (61a) and (61b). We, therefore, have shown that, in addition to the often-studied two-photon resonance trapping, the damping system can also be prepared in a multiphoton coherent state which is physically decoupled from the irreversible loss mechanism inherited by the common level 2 .

\section{Case study of two-photon quasitrapping}

In this subsection we shall present our results for $\Lambda$ three-level system, cf. Fig. 1(b), irradiated by two strong laser fields. The three levels of the system are designated as $E_{1}=0.0, E_{2}=1000.0$, and $E_{3}=900.0$, with arbitrary units being adopted. Parities of levels 1 and 3 are the same, and are opposite to that of the level 2. Furthermore, the second, i.e., the common, level is characterized by an irreversible loss rate $g_{2}$, equal to 0.4 in this case. Only cases of two-photon processes will be discussed in the following; cases of other multiphoton processes (i.e., more than two photon), nonetheless, reveal similar outcomes to those of the two-photon ones. All calculations made here are based on the $3 \times 3$ effective non-Hermitian Hamiltonian $\hat{A}_{3}$ defined in Eq. (44); comparisons with the numerically exact, i.e., based on the truncated, but converged, total Floquet Hamiltonian $\hat{A}_{F}$ of Eq. (11), results are made when necessary.

In Figs. 2(a) and 2(b) we show, respectively, the real and imaginary parts of the quasienergies $\lambda_{100}, \lambda_{2,-10}$, and $\lambda_{3,-1,+1}$ as functions of the frequency $\omega_{2}$ of the second laser at parameters $\omega_{1}=995.0, b_{i}^{(\alpha \beta)}=5.0$ for all $i=1,2$ and $(\alpha \beta)=(12),(23)$. Here we have chosen frequencies $\omega_{1}$ and $\omega_{2}$ such that the first field mainly drives the $1 \rightarrow 2$ transition, while the second field is for the $2 \rightarrow 3$ transition. The real parts exhibit the well-known structure of avoided crossings nearby $\omega_{2} \approx\left|E_{3}-E_{2}\right|$ and $\omega_{1}-\omega_{2} \approx\left|E_{3}-E_{1}\right|$, and dictate the oscillations of the system among levels when evolving. We note that the strengths of laser fields are rather large, therefore regions of avoided crossing are quite extended and splittings between quasienergy levels at crossings are large, on the order of magnitude of the corresponding Rabi frequencies $b_{1}^{(12)}$ and $b_{2}^{(23)}$. While two of the imaginary parts of 

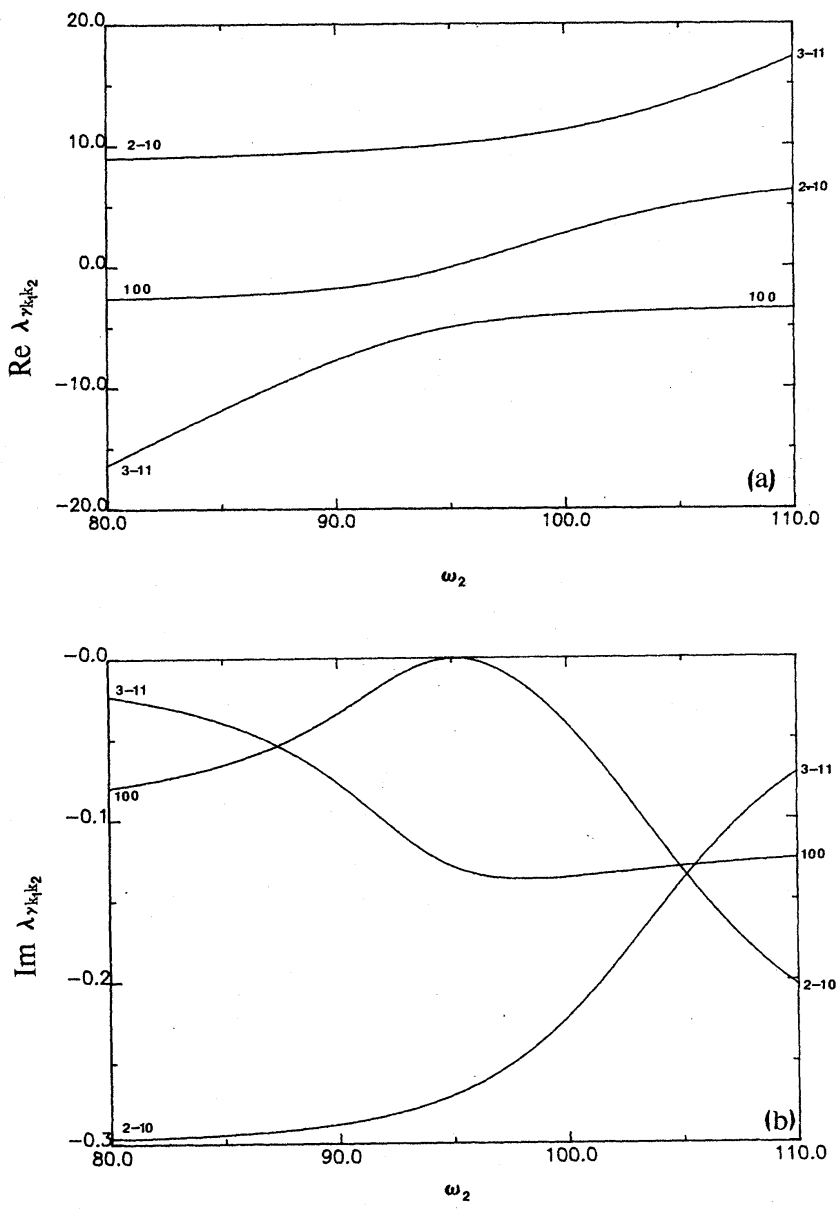

FIG. 2. (a) Real and (b) imaginary parts of the quasienergies as functions of the frequency $\omega_{2}$ at $E_{2}-E_{1}=1000.0$ (arbitrary units) $, \quad E_{2}-E_{3}=100.0, \quad \omega_{1}=995.0, \quad b_{1}^{(12)}=b_{1}^{(23)}=b_{2}^{(23)}=b_{2}^{(12)}$ $=5.0$, and $g_{2}=0.4$ of the $\Lambda$-type three-level system.

quasienergies remain on the order of magnitude of the loss rate $g_{2}$ of the second level over all of the range of the frequencies $\omega_{2}$, i.e., from 80.0 to 110.0 as presented, the third one is vanishingly small around the location of the two-photon resonance, i.e., when $\omega_{2} \approx 95.0$ in this case. In Fig. 3(a) we study more closely this third imaginary part near the two-photon resonance, i.e., for $\omega_{2}$ ranging from 94.5 to 95.5 , for a wide range of the Rabi frequencies $b_{2}^{(12)}=b_{2}^{(23)}$ at fixed $\omega_{1}=995.0$ and fixed $b_{1}^{(12)}$ $=b_{1}^{(23)}=5.0$. We easily found that (i) this third imaginary component is small, but never identically zero, over a large region of $\omega_{2}$; (ii) the maximum position, or the minimum in magnitude, of the imaginary concaving curve, as a function of $\omega_{2}$, shifts away from the exact two-photon resonance condition $\omega_{1}-\omega_{2}=\left|E_{3}-E_{1}\right|$ and toward larger $\omega_{2}$ as the Rabi frequencies $b_{2}^{(12)}=b_{2}^{(23)}$ are increased [see also Fig. 3(b)] manifesting the effect of the antirotating mechanism; (iii) the magnitude of the concaving curve gradually increases, although it still stays small, as we increase the quantities $b_{2}^{(12)}$ and $b_{2}^{(23)}$; and, finally, (iv) the shape of the concaving curve is flatter at smaller, say 1.0 , and larger, say $10.0, b_{2}^{(12)}$ and $b_{2}^{(23)}$, but steeper in
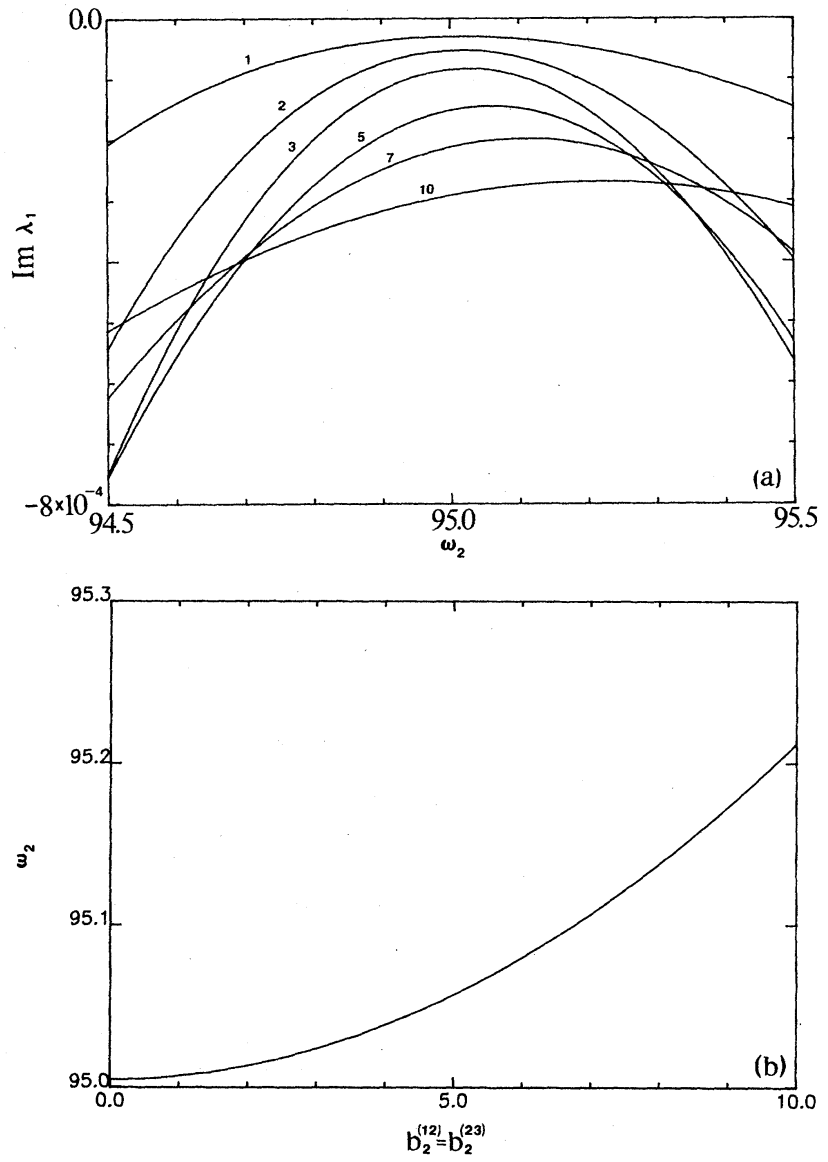

FIG. 3. (a) Imaginary part of the quasienergy having the smallest imaginary energy nearby the two-photon resonance condition for the second Rabi frequencies $b_{2}^{(12)}=b_{2}^{(23)}=1.0,2.0$, $3.0,5.0,7.0$, and 10.0, respectively, at the fixed parameters $b_{1}^{(12)}=b_{1}^{(23)}=5.0, \omega_{1}=995.0$, and $g_{2}=0.4 ;$ (b) the location of the minimum, in magnitude, of the smallest imaginary part of the quasienergy as a function of the second Rabi frequency $b_{2}^{(12)}=b_{2}^{(23)}$ at the fixed parameters $\omega_{1}=995.0$, $b_{1}^{(12)}=b_{1}^{(23)}=5.0$, and $g_{2}=0.4$.

between, say 3.0 and 5.0. The above observations are important in that the exact trapping at the exact two-photon resonance concluded in the RWA limit does not exist when non-RWA effects, the antirotating effect in this case, are taken into account. But, nevertheless, we can still have quasitrapping due to the small magnitude of the third imaginary part of quasienergies.

Time-dependent transition probabilities of finding the system in its various levels, assuming the system initially lies in the level 1 , are shown in Fig. $4(a)$ at $b_{1}^{(12)}$ $=b_{1}^{(23)}=b_{2}^{(12)}=b_{2}^{(23)}=5.0, \omega_{1}=995.0$, and $\omega_{2}=95.0569$, corresponding to a maximum in Fig. 3(a). The nondamping, i.e., $g_{2}=0$, counterparts of Fig. 4(a) are presented in Fig. 4(b) for easy comparisons. Figures 5(a) and 5(b) show the good agreement between the calculations from the approximate $\hat{A}_{3}$ of Eq. (44) and from the exact $\hat{A}_{F}$ of Eq. (11) for the nondamping case. From Fig. 4(a) we readily see that while the population probability $P_{12}(t)$ of finding the system in the second level damps away quickly, those 


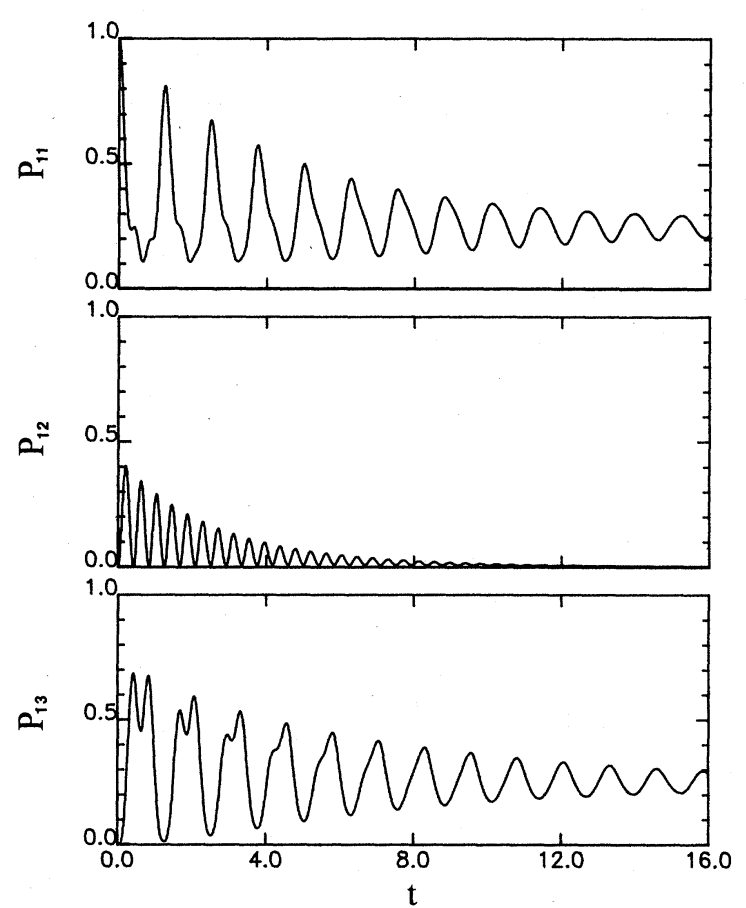

(a)

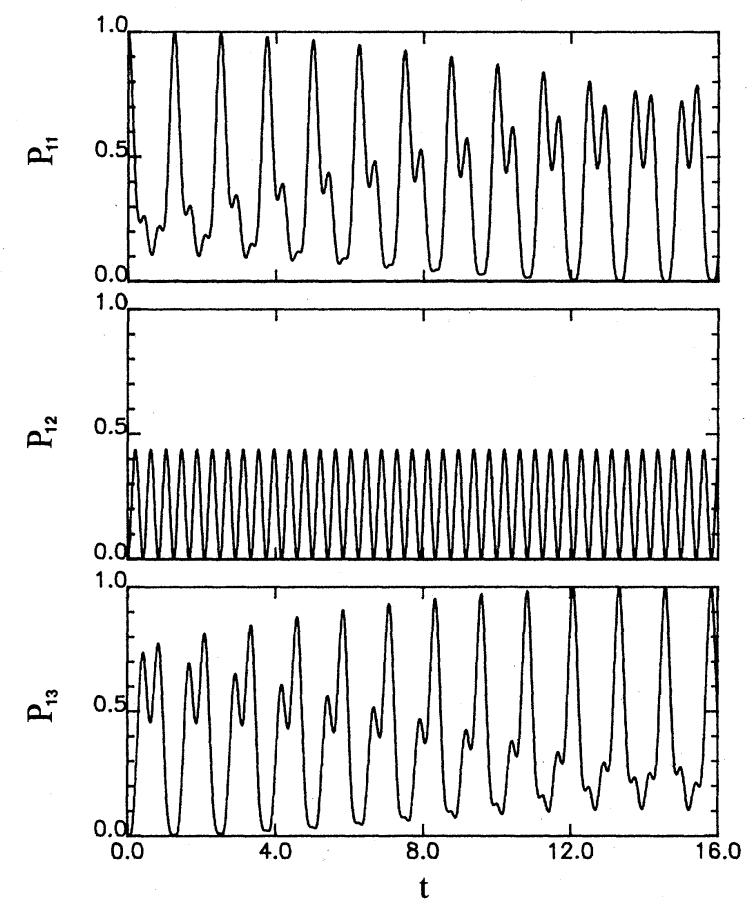

(b)

FIG. 4. Time-dependent transition probabilities $P_{11}(t)$, $P_{12}(t)$, and $P_{13}(t)$, as functions of time $t$ for (a) the dissipative three-level system characterized by a loss rate $g_{2}=0.4$, and (b) the nondamping system at the parameters $\omega_{1}=995.0$, $\omega_{2}=95.0569$, and $b_{1}^{(12)}=b_{1}^{(23)}=b_{2}^{(12)}=b_{2}^{(23)}=5.0$.

of levels 1 and $3, P_{11}(t)$ and $P_{13}(t)$, respectively, converge oscillatorily to some finite values at some large times. Although $P_{11}(t)$ and $P_{13}(t)$ will stay at some finite values

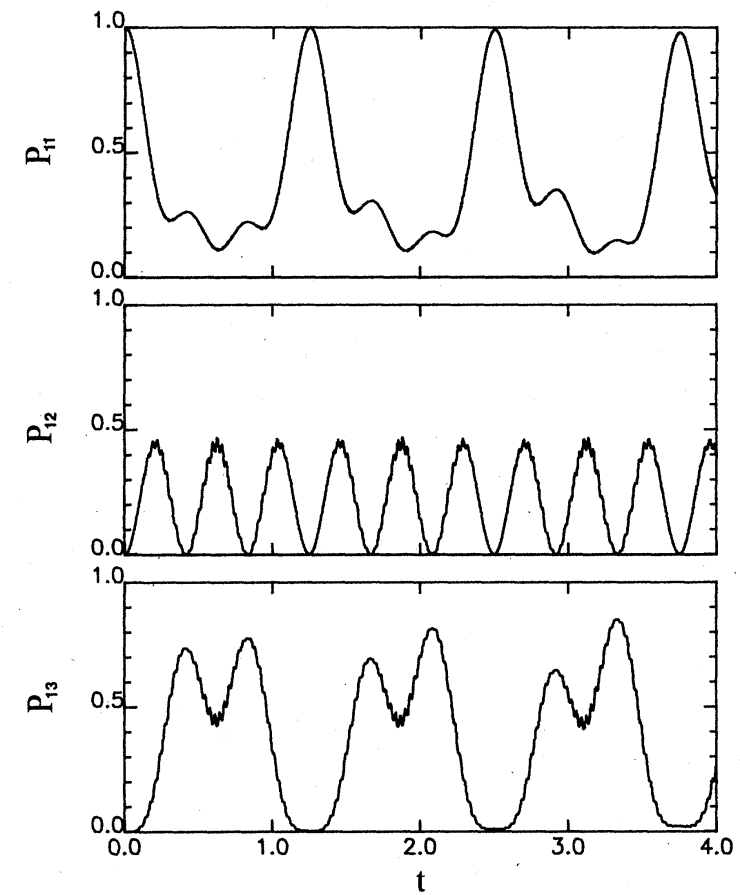

(a)

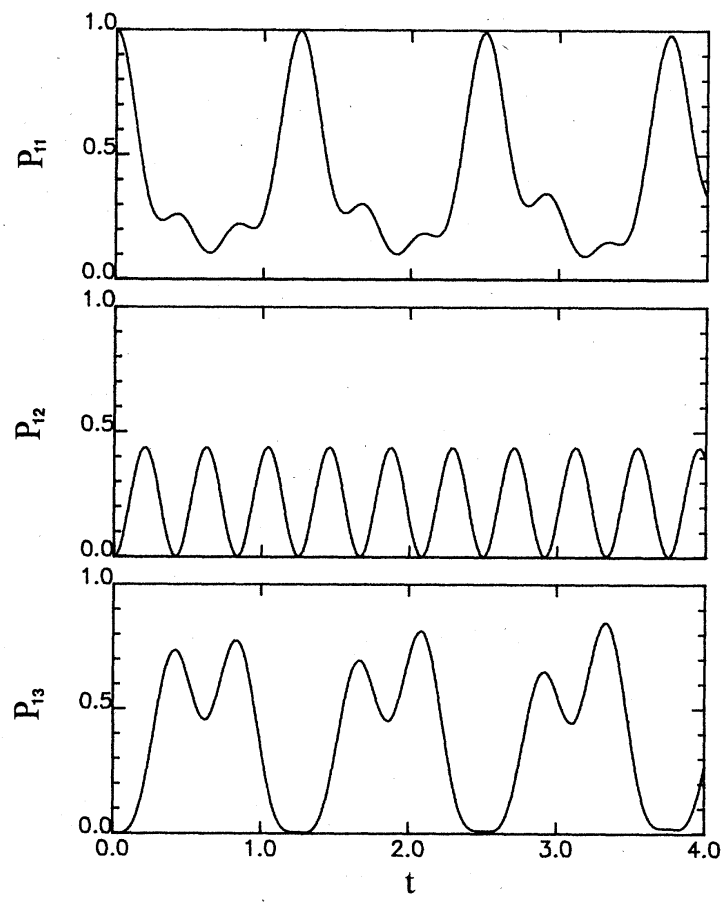

(b)

FIG. 5. Comparison between (a) the exact and (b) the approximate $\hat{A}_{3}$ results of the time-dependent transition probabilities $P_{11}(t), P_{12}(t)$, and $P_{13}(t)$ for the nondamping three-level system at the same conditions as depicted in Fig. 4 . The exact results were obtained from a truncated, but converged, Floquet Hamiltonian.

for some large durations of time, it will eventually drop to zero because of the nonvanishing, but small, third imagi- 
nary part of the quasienergies discussed in Fig. 3(a). This can be comprehended by displaying $P_{11}(t), P_{13}(t)$, and $P_{12}(t)$ as functions of $\omega_{2}$ at various instants of time. In Figs. 6(a) and 6(b) $P_{11}(t)$ and $P_{13}(t)$, respectively, are shown as functions of $\omega_{2}$ at $t=20.0,50.0,200.0$, and 1000.0. The profiles of both $P_{11}(t)$ and $P_{13}(t)$ peak around the two-photon resonance condition, i.e., $\omega_{2} \approx \omega_{1}-\left|E_{3}-E_{1}\right|$, and become narrower and lower as we increase the observation time. The shrinkage of the peaks indicates that the system is not exactly trapped in a coherently superposing state of levels 1 and 3, but rather quasitrapped. Figures 6(c) and 6(d) show $P_{12}(t)$ as a function of $\omega_{2}$ at the same instants of time as in Figs. 6(a) and 6(b). Two features are observed for $P_{12}(t)$, one is that the amplitude of the $P_{12}(t)$ decreases rapidly, compared with that of $P_{11}(t)$ or $P_{13}(t)$ in Figs. 6(a) or 6(b), as the time passes by, and the other is that a dip, which becomes narrower as the time is increased, is formed around the twophoton resonance condition. Also clearly seen is the small displacement of the bottom of the dip away from the exact two-photon resonance frequency, i.e., $\omega_{2}=95.0$ in this case, due to the antirotating effect. The dip is apparently caused by the quasitrapping of the system in levels 1 and 3 , and explains the observation of dark lines recently reported by several groups. ${ }^{11}$

\section{DYNAMICAL EVOLUTION OF THE SU(3) COHERENCE VECTOR OF DISSIPATIVE THREE-LEVEL SYSTEMS}

The time evolution of a dissipationless $N$-level system can be described by the rotation of an $\left(N^{2}-1\right)$ dimensional coherence vector, of constant length, based on the group-SU $(N)$ generators. ${ }^{7}$ In a previous paper ${ }^{4}$ we have used the MMFT-GVV approach to the study of the dynamical behavior of dissipationless three-level systems in the presence of intense bichromatic fields in terms of an eight-dimensional coherence vector $\mathbf{S}(t)$. In this section we extend the $S U(3)$ study to the three-level systems with dissipative terms. As the system evolves in response to external bichromatic fields, its dynamical behavior is mirrored in the geometrical motions of $\mathbf{S}(t)$-a shrinking rotating vector. In the case of resonance (quasi-) trapping, the shrinkage of the coherence vector will gradually become very slow or even stopped. Detailed analytical and pictorial study of this phenomenon will be exploited in this section.

Following closely the formulation of the equation of motion of a nondamping coherent vector, we can at first express the total Hamiltonian of Eq. (1) and the corresponding density matrix operator in terms of the eight (traceless) $\mathrm{SU}(3)$ generators $\left\{\hat{y}_{i} \mid i=1,2, \ldots, 8\right\}$ (to be given explicitly later),

$$
\begin{aligned}
& \hat{H}=\frac{1}{3}\left[\sum_{\alpha=1}^{3} E_{\alpha}\right] \widehat{I}+\frac{1}{2} \sum_{j=1}^{8} \Gamma_{j}(t) \hat{s}_{j}, \\
& \widehat{G}=\frac{1}{3}\left(\sum_{\alpha=1}^{3} g_{\alpha}\right] \widehat{I}+\frac{1}{2} \sum_{j=1}^{8} \Lambda_{j} \hat{s}_{j},
\end{aligned}
$$

and
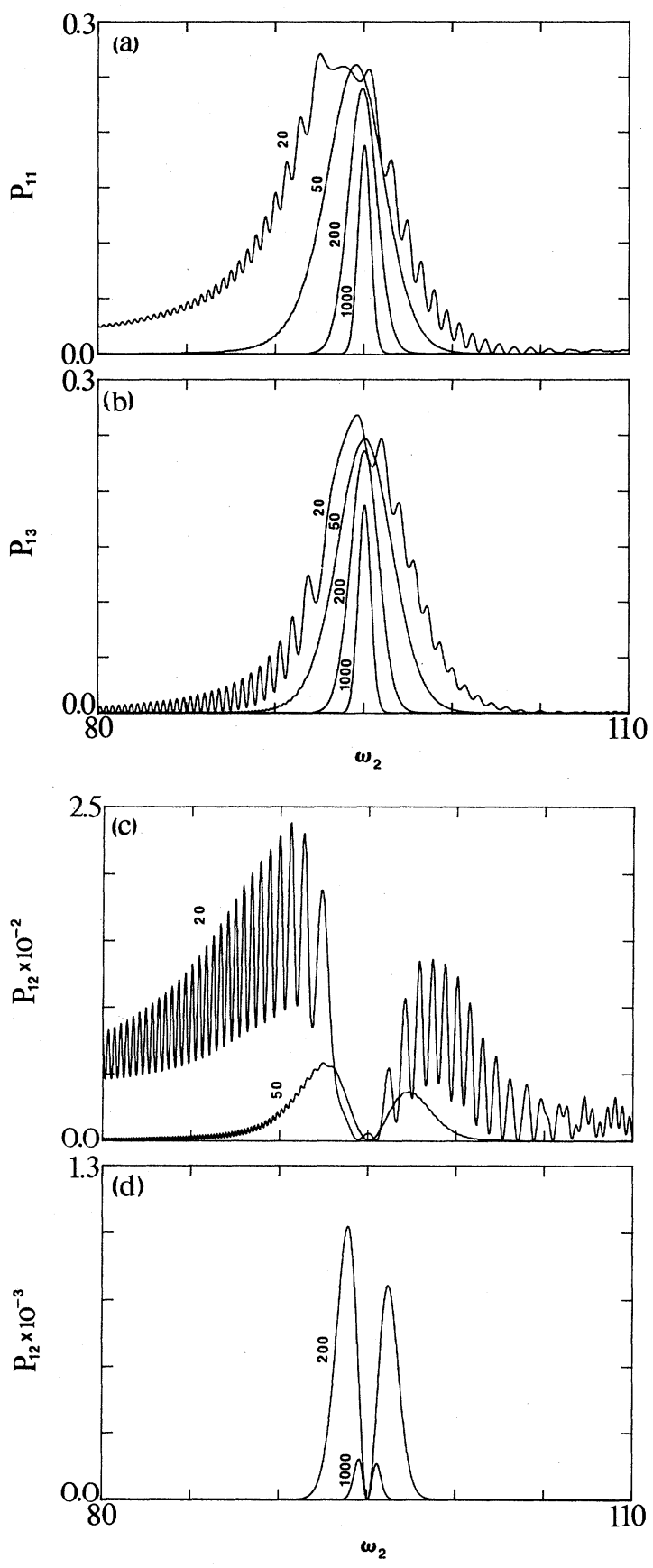

FIG. 6. The time-dependent transition probabilities (a) $P_{11}(t)$, (b) $P_{13}(t)$, and (c) and (d) $P_{12}(t)$ as functions of the second laser frequency $\omega_{2}$ for $t=20.0,50.0,200.0$, and 1000.0, respectively, at the same conditions as depicted in Fig. 2.

$$
\hat{\rho}(t)=\frac{1}{3} S_{0}(t) \hat{I}+\frac{1}{2} \sum_{j=1}^{8} S_{j}(t) \hat{s}_{j}
$$

where quantities $E_{\alpha}$ 's and $g_{\alpha}$ 's have been defined in Sec. II, and coefficients $\Gamma_{j}(t), \Lambda_{j}, S_{0}(t)$, and $S_{j}(t)$, with $j=1, \ldots, 8$, are defined as

$$
\Gamma_{j}(t)=\operatorname{Tr}\left[\hat{H}(t) \hat{s}_{j}\right],
$$




$$
\begin{aligned}
& \Lambda_{j}=\operatorname{Tr}\left(\hat{G} \hat{\Sigma}_{j}\right), \\
& S_{0}(t)=\left[\hat{\rho}_{11}(t)+\hat{\rho}_{22}(t)+\hat{\rho}_{33}(t)\right],
\end{aligned}
$$

and

$$
S_{j}(t)=\operatorname{Tr}\left[\hat{\rho}(t) \hat{s}_{j}\right] .
$$

Coefficient $S_{0}(t)$ of Eq. (68) is introduced to make room for the irreversible population loss of the system into its surroundings. The equation of motion for the damping coherence vector $\mathbf{S}(t) \equiv\left(S_{1}(t), S_{2}(t), \ldots, S_{8}(t)\right)$ can be obtained by substituting Eqs. (66) -(68) into the Liouville equation

$$
i \frac{d \hat{\rho}(t)}{d t}=[\hat{H}(t), \hat{\rho}]_{-}-i\{\hat{G}, \hat{\rho}\}_{+},
$$

where

$$
[\widehat{A}, \widehat{B}]_{-} \equiv \hat{A} \widehat{B}-\widehat{B} \hat{A} \text { and }\{\widehat{A}, \widehat{B}\}_{+} \equiv \widehat{A} \widehat{B}+\widehat{B} \hat{A} .
$$

This gives

$$
\begin{aligned}
\frac{d \mathbf{S}_{l}(t)}{d t}= & \sum_{j, k} f_{l j k} \Gamma_{j}(t) \mathbf{S}_{k}(t) \\
& -\left[\sum_{j, k} d_{l j k} \Lambda_{l} S_{k}(t)\right. \\
& \left.+\frac{2}{3}\left[\sum_{\alpha=1}^{3} g_{\alpha}\right] \mathbf{S}_{l}(t)+\frac{2}{3} \Lambda_{l} S_{0}(t)\right],
\end{aligned}
$$

where the first summation on the righ-hand side of Eq. (74) causes the coherence vector $S$ to rotate with a generalized angular frequency, and terms inside the large parentheses are frictions experienced by the coherence vector $\mathbf{S}(t)$. The two structure constants $f_{l j k}$ and $d_{l j k}$ are defined as

$$
f_{l j k} \equiv-\frac{1}{4} i \operatorname{Tr}\left(\hat{s}_{l}\left[\hat{s}_{j}, \hat{z}_{k}\right]\right),
$$

which is antisymmetric under the permutation of any two indices, and

$$
d_{l j k} \equiv \frac{1}{4} \operatorname{Tr}\left(\hat{\Sigma}_{l}\left\{\hat{z}_{j}, \hat{z}_{k}\right\}_{+}\right),
$$

is symmetric under permutations. The nonvanishing SU(3) structure constants $f_{l j k}$ and $d_{l j k}$ are listed in Table I. The equation of motion for the coefficient $S_{0}(t)$ can be written as

$\frac{d S_{0}(t)}{d t}=-\frac{2}{3}\left(\sum_{\alpha=1}^{3} g_{\alpha}\right] S_{0}(t)-\frac{1}{4} \sum_{j, k} \operatorname{Tr}\left(\left\{\hat{\Sigma}_{j}, \hat{\Sigma}_{k}\right\}_{+}\right) \Lambda_{j} S_{k}(t)$,

In the special cases considered in Sec. III we have ap-

\begin{tabular}{|c|c|c|c|c|}
\hline$j k$ & $k l$ & $d_{j k l}$ & $j k l$ & $f_{j k l}$ \\
\hline 11 & 18 & $-1 / \sqrt{3}$ & 123 & 1 \\
\hline 14 & 46 & $1 / 2$ & 145 & $-1 / 2$ \\
\hline 15 & 57 & $1 / 2$ & 167 & $-1 / 2$ \\
\hline 22 & 28 & $-1 / \sqrt{3}$ & 246 & $1 / 2$ \\
\hline 24 & 45 & $1 / 2$ & 257 & $-1 / 2$ \\
\hline 26 & 67 & $-1 / 2$ & $\begin{array}{lll}347\end{array}$ & $-1 / 2$ \\
\hline 33 & 38 & $-1 / \sqrt{3}$ & 356 & $-1 / 2$ \\
\hline 34 & 44 & $1 / 2$ & 478 & $\sqrt{3} / 2$ \\
\hline 35 & 55 & $-1 / 2$ & 568 & $-\sqrt{3} / 2$ \\
\hline 36 & 66 & $-1 / 2$ & & \\
\hline 37 & 77 & $1 / 2$ & & \\
\hline 44 & 48 & $1 / 2 \sqrt{3}$ & & \\
\hline 55 & 58 & $1 / 2 \sqrt{3}$ & & \\
\hline 66 & 68 & $1 / 2 \sqrt{3}$ & & \\
\hline 77 & 78 & $1 / 2 \sqrt{3}$ & & \\
\hline 88 & 88 & $1 / \sqrt{3}$ & & \\
\hline
\end{tabular}
proximated the time-dependent total Hamiltonian $\widehat{H}(t)-i \widehat{G}$ of Eq. (1) by a $3 \times 3$ effective time-independent non-Hermitian Hamiltonian $\hat{A}_{3}$ of Eq. (38) in the properly chosen transformed frame defined by Eqs. (40) and (41). The matrix $\hat{A}_{3}$ of Eq. (44), i.e., the two-photon trapping Hamiltonian, can be partitioned into an unperturbed part $\hat{A}_{30}$ [Eq. (49)] and a perturbation term $\hat{V}_{3}$ [Eq. (50)], where $\hat{A}_{30}$ is the RWA Hamiltonian at two-photon reso-
TABLE I. Nonvanishing SU(3) structure constants $f_{j k l}$ and $d_{j k l}$

nance. Similarly, the multiphoton effective Hamiltonian, Eq. (59), can be divided into a RWA-like unperturbed Hamiltonian and a perturbation term. As the dynamical behavior is dominated by the $\widehat{A}_{30}$ Hamiltonian, we can thus introduce the following Hioe-Eberly-type SU(3) generators for the study of the two-photon and multiphoton dynamics of dissipative three-level systems:

$$
\begin{aligned}
& \widehat{s}_{1}=\frac{1}{b}\left[\begin{array}{ccc}
0 & \alpha_{n_{1}} & 0 \\
\alpha_{n_{1}} & 0 & \beta_{n_{2}} \\
0 & \beta_{n_{2}} & 0
\end{array}\right] \text {, } \\
& \hat{\jmath}_{2}=\frac{i}{b}\left[\begin{array}{ccc}
0 & \alpha_{n_{1}} & 0 \\
-\alpha_{n_{1}} & 0 & -\beta_{n_{2}} \\
0 & \beta_{n_{2}} & 0
\end{array}\right] \text {, } \\
& \hat{s}_{3}=\frac{1}{b^{2}}\left[\begin{array}{ccc}
\alpha_{n_{1}}^{2} & 0 & \alpha_{n_{1}} \beta_{n_{2}} \\
0 & -b^{2} & 0 \\
\alpha_{n_{1}} \beta_{n_{2}} & 0 & \beta_{n_{2}}^{2}
\end{array}\right] \text {, } \\
& \widehat{s}_{4}=i\left[\begin{array}{ccc}
0 & 0 & -1 \\
0 & 0 & 0 \\
1 & 0 & 0
\end{array}\right] \text {, } \\
& \hat{3}_{5}=\frac{1}{b}\left[\begin{array}{ccc}
0 & \beta_{n_{2}} & 0 \\
\beta_{n_{2}} & 0 & -\alpha_{n_{1}} \\
0 & -\alpha_{n_{1}} & 0
\end{array}\right] \text {, } \\
& \hat{\jmath}_{6}=\frac{i}{b}\left[\begin{array}{ccc}
0 & -\beta_{n_{2}} & 0 \\
\beta_{n_{2}} & 0 & -\alpha_{n_{1}} \\
0 & \alpha_{n_{1}} & 0
\end{array}\right] \text {, }
\end{aligned}
$$




$$
\hat{s}_{7}=\frac{1}{b^{2}}\left[\begin{array}{ccc}
2 \alpha_{n_{1}} \beta_{n_{2}} & 0 & \beta_{n_{2}}^{2}-\alpha_{n_{1}}^{2} \\
0 & 0 & 0 \\
\beta_{n_{2}}^{2}-\alpha_{n_{1}}^{2} & 0 & -2 \alpha_{n_{1}} \beta_{n_{2}}
\end{array}\right],
$$

and

$$
\hat{s}_{8}=\frac{1}{\sqrt{3} b^{2}}\left[\begin{array}{ccc}
2 \beta_{n_{2}}^{2}-\alpha_{n_{1}}^{2} & 0 & -3 \alpha_{n_{1}} \beta_{n_{2}} \\
0 & -b^{2} & 0 \\
-3 \alpha_{n_{1}} \beta_{n_{2}} & 0 & 2 \alpha_{n_{1}}^{2}-\beta_{n_{2}}^{2}
\end{array}\right]
$$

$$
\frac{d}{d t}\left[\begin{array}{l}
S_{1} \\
S_{2} \\
S_{3} \\
S_{8} \\
S_{0}
\end{array}\right]=\left[\begin{array}{ccccc}
-g_{2} & -\Delta & 0 & 0 & 0 \\
\Delta & -g_{2} & 2 b & 0 & 0 \\
0 & -2 b & -g_{2} & -\frac{1}{\sqrt{3}} g_{2} & \frac{2}{3} g_{2} \\
0 & 0 & -\frac{1}{\sqrt{3}} g_{2} & -\frac{1}{3} g_{2} & \frac{2}{3 \sqrt{3}} g_{2} \\
0 & 0 & g_{2} & \frac{1}{\sqrt{3}} g_{2} & -\frac{2}{3} g_{2}
\end{array}\right]\left[\begin{array}{l}
S_{1} \\
S_{2} \\
S_{3} \\
S_{8} \\
S_{0}
\end{array}\right]
$$

where

$$
b=\left(\alpha_{n_{1}}^{2}+\beta_{n_{2}}^{2}\right)^{1 / 2} .
$$

In the following we shall first consider the solution of the coherence vector corresponding to the RWA Hamiltonian $\hat{A}_{30}$, Eq. (44), at the two-photon resonance trapping condition. It can be readily shown that the nine coupled equations of motion of $\mathbf{S}(t)$, Eq. (74), and $S_{0}(t)$, Eq. (77), can be factored into two independent sets of equations of motion of dimensions five and four, respectively:

$S_{5}(t)=(\alpha \beta / \Omega)\left[\left(\lambda_{2} A_{2}+\lambda_{3} A_{3}\right)+\right.$ c.c. $]$,

$S_{6}(t)=i(\alpha \beta / \Omega)\left[\left(\lambda_{2} A_{2}+\lambda_{3} A_{3}\right)-\right.$ c.c. $]$,

$$
\frac{d}{d t}\left[\begin{array}{l}
S_{4} \\
S_{5} \\
S_{6} \\
S_{7}
\end{array}\right]=\left[\begin{array}{cccc}
0 & -b & 0 & 0 \\
b & -g_{2} & \Delta & 0 \\
0 & -\Delta & -g_{2} & -b \\
0 & 0 & b & 0
\end{array}\right]\left[\begin{array}{l}
S_{4} \\
S_{5} \\
S_{6} \\
S_{7}
\end{array}\right]
$$

where $\Delta=\left(E_{2}-E_{1}\right)-\omega_{1}$ and, $b=\left(\alpha^{2}+\beta^{2}\right)^{1 / 2}$, with $\alpha=b_{1}^{(12)}$ and $\beta=\beta_{2}^{(23)}$. In the following, without loss of generalization, we shall assume that the system is initially in the ground level $|1\rangle$, and that the field phases $\phi_{1}=\phi_{2}=0$. The coherence vector $s_{i}(t), i=1,2, \ldots, 8$, can be solved exactly, in the RWA limit, in terms of the three quasienergy eigenvalues $\lambda_{i}(i=1,2,3)$ of $\hat{A}_{30}$ [cf. Eqs. (52a) and (52b)], namely,

$$
\begin{aligned}
& \lambda_{1}=0, \\
& \lambda_{2}=\frac{1}{2}\left(\Delta-i g_{2}\right)+\frac{1}{2}\left[\left(\Delta-i g_{2}\right)^{2}+4 \Omega^{2}\right]^{1 / 2},
\end{aligned}
$$

and

$$
\lambda_{3}=\frac{1}{2}\left(\Delta-i g_{2}\right)-\frac{1}{2}\left[\left(\Delta-i g_{2}\right)^{2}+4 \Omega^{2}\right]^{1 / 2},
$$

where $\Omega=b=\left(\alpha^{2}+\beta^{2}\right)^{1 / 2}$. (Note that $\Omega^{2}=-\lambda_{2} \lambda_{3}$.) The analytical solutions for $S_{i}(t)$ are given by

$$
\begin{gathered}
S_{1}(t)=\Omega \alpha^{2}\left[\left(\lambda_{2} A_{2}+\lambda_{3} A_{3}\right)\left(A_{2}^{*}+A_{3}^{*}\right)+\text { c.c. }\right], \\
S_{2}(t)=-i \Omega \alpha^{2}\left[\left(\lambda_{2} A_{2}+\lambda_{3} A_{3}\right)\left(A_{2}^{*}+A_{3}^{*}\right)-\text { c.c. }\right], \\
S_{3}(t)=\alpha^{2}\left\{\left(\Omega^{2}-\left|\lambda_{2}\right|^{2}\right) B_{2}+\left(\Omega^{2}-\left|\lambda_{3}\right|^{2}\right) B_{3}\right. \\
\left.\quad+\left[\left(\Omega^{2}-\lambda_{2} \lambda_{3}^{*}\right) B_{23}+\text { c.c. }\right]\right\}, \\
S_{4}(t)=i\left(\alpha^{3} \beta / \Omega^{2}\right)\left[\left(A_{2}+A_{3}\right)-\text { c.c. }\right],
\end{gathered}
$$

$S_{7}(t)=\frac{4 \alpha^{2} \beta\left(\alpha^{2}-\beta^{2}\right)}{\Omega^{2}}\left(B_{2}+B_{3}\right)+\alpha \beta\left[\left(A_{2}+A_{3}\right)+\right.$ c.c. $]$,

and

$$
\begin{gathered}
S_{8}(t)=\frac{2}{\sqrt{3}} \frac{\beta^{2}}{\Omega^{2}}-\frac{\alpha^{2}}{\sqrt{3}}\left\{\left(\Omega^{2}+\left|\lambda_{2}\right|^{2}\right) B_{2}+\left(\Omega^{2}+\left|\lambda_{3}\right|^{2}\right) B_{3}\right. \\
\left.+\left[\left(\Omega^{2}+\lambda_{2} \lambda_{3}^{*}\right) B_{23}+\text { c.c. }\right]\right\},
\end{gathered}
$$

where

$$
\begin{aligned}
& A_{k}=\exp \left(-i \lambda_{k} t\right) /\left(\Omega^{2}+\lambda_{k}^{2}\right) \quad(k=2,3), \\
& B_{k}=\exp \left[-i\left(\lambda_{k}-\lambda_{k}^{*}\right) t\right] /\left|\Omega^{2}+\lambda_{k}^{2}\right|^{2} \quad(k=2,3),
\end{aligned}
$$

and

$$
B_{23}=\exp \left[-i\left(\lambda_{2}-\lambda_{3}^{*}\right) t\right] /\left[\left(\Omega^{2}+\lambda_{2}^{2}\right)\left(\Omega^{2}+\lambda_{3}^{* 2}\right)\right] .
$$

Note that in the limit $t \rightarrow \infty$,

$$
S_{i}\left(t_{\infty}\right) \rightarrow 0, \quad i=1,2,3, \ldots, 7
$$

but

$$
S_{8}\left(t_{\infty}\right) \rightarrow 2 \beta^{2} / \sqrt{3} \Omega^{2}
$$

Thus at exact two-photon resonance condition, in the RWA limit, the eight-dimensional coherent vector eventually evolves to a one-dimensional scalar and the population is trapped in the $S_{8}$ component only. It is interesting to note that the population scalar $S_{0}(t)\left(\equiv \rho_{11}+\rho_{22}+\rho_{33}\right)$ 


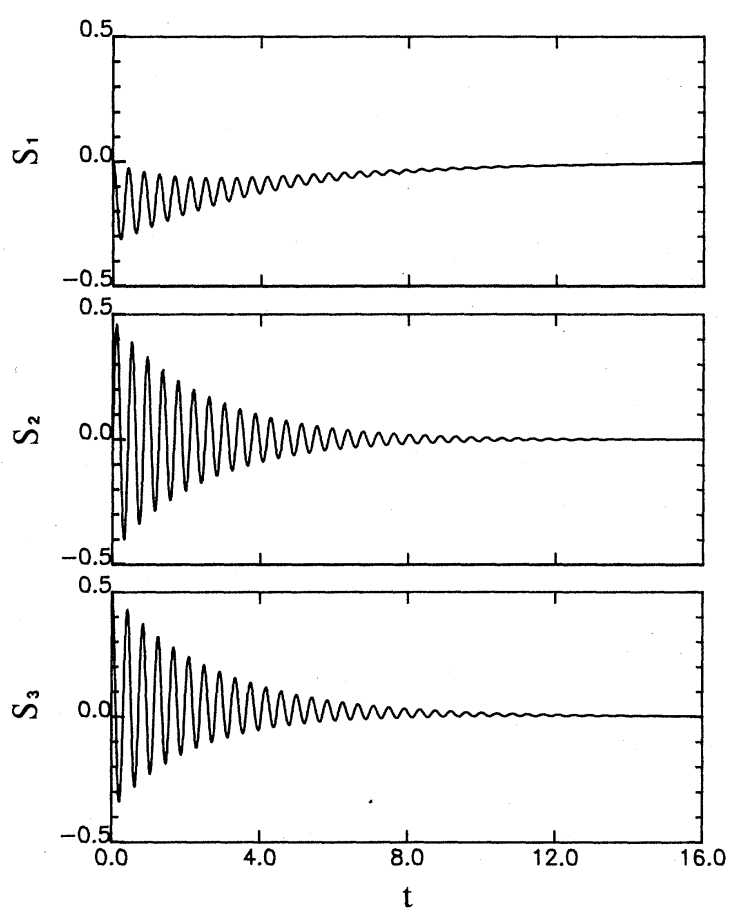

(a)
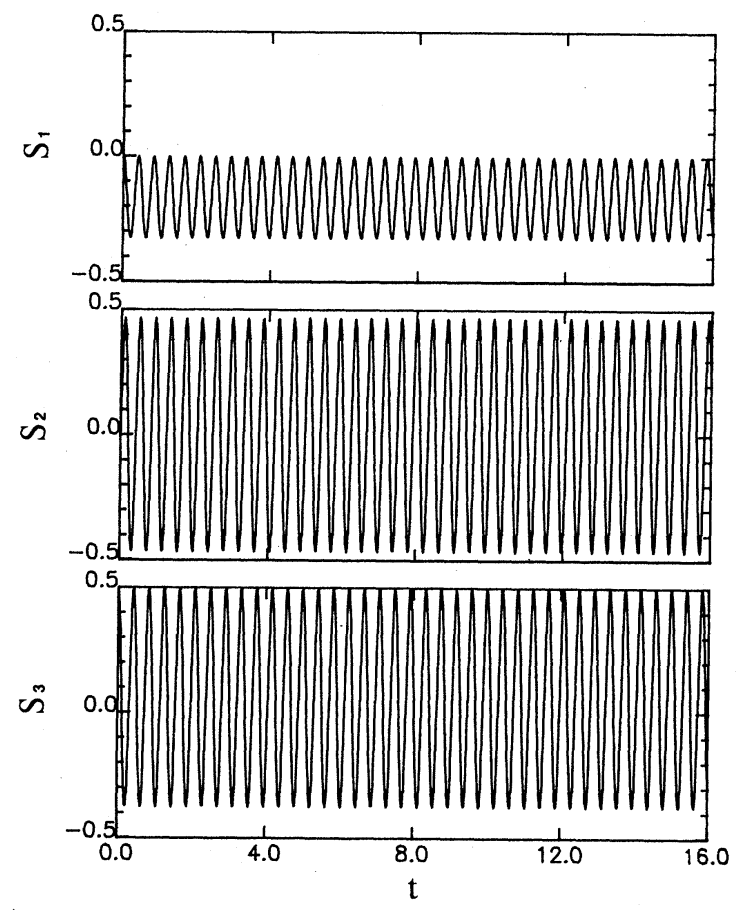

(b)

FIG. 7. The first three components of the coherence vector, $S_{1}(t), S_{2}(t)$, and $S_{3}(t)$, as functions of time for (a) the dissipative three-level system, and (b) the nondamping system at the same conditions depicted in Fig. 4.

in this case reduces to

$$
S_{0}\left(t_{\infty}\right) \rightarrow \frac{\sqrt{3}}{2} S_{8}\left(t_{\infty}\right)=\frac{\beta^{2}}{\Omega^{2}},
$$
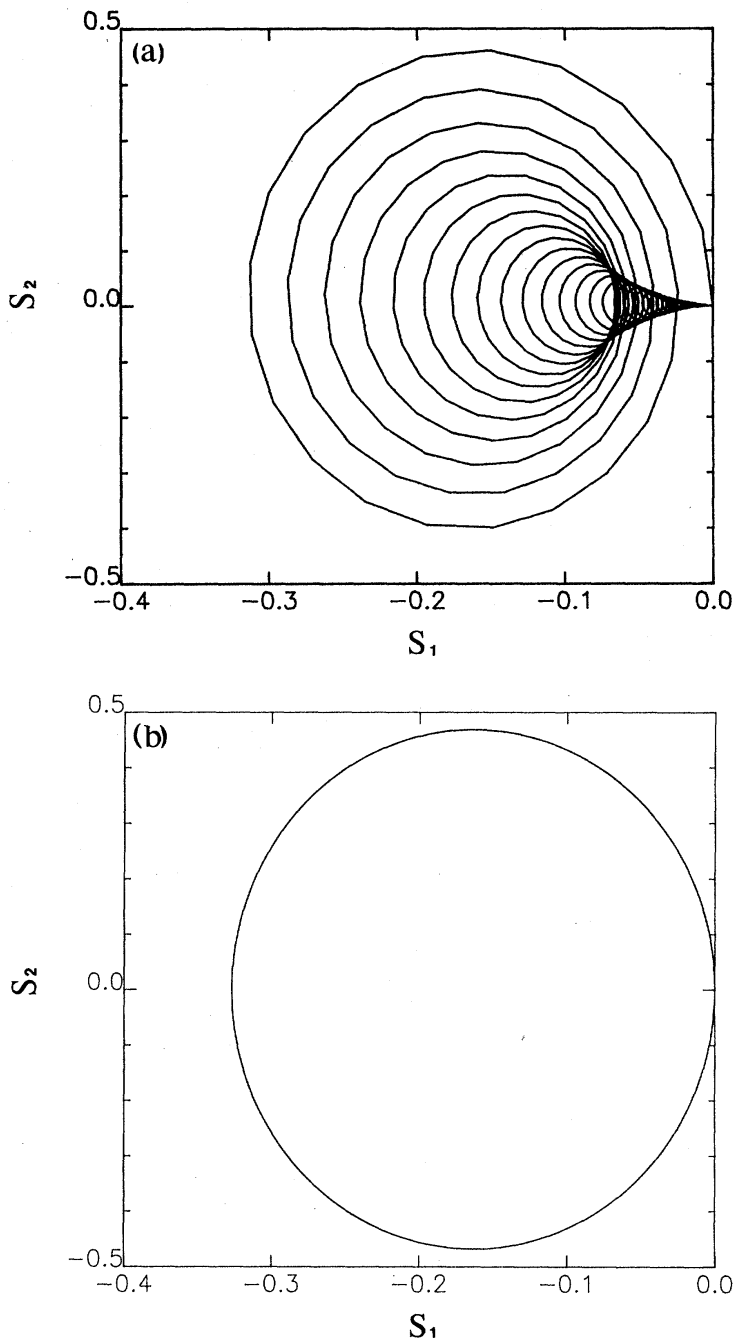

FIG. 8. Projection of the trajectory of the coherence vector on the $S_{1}-S_{2}$ plane for the same duration as shown in Fig. 7 for (a) the dissipative three-level system and (b) the nondamping system.

and both $S_{8}$ and $S_{0}$ are independent of the damping constant $g_{2}$.

We now return to the full effective Hamiltonian $\widehat{A}_{3}=\widehat{A}_{30}+\widehat{V}_{3}$, Eq. (44). The inclusion of the perturbation term $\hat{V}_{3}$ modifies the RWA trapping condition, leading to the quasitrapping phenomenon as described in Sec. III B.

The nine coupled equations for $S_{i}(t), i=0,1,2, \ldots, 8$, are no longer factorizable into two independent sets of equations of smaller dimensions as shown in Eqs. (79) and (80). The solutions of these nine coupled equations become intractable analytically but can be solved numerically in principle, though tedious and time-consuming, subject to proper initial conditions for $\mathbf{S}(t=0)$ and $S_{0}(t=0)$. As pointed out in our previous paper, ${ }^{4}$ instead of involving these time-dependent generalized Bloch equations directly, the coherent vector $\mathbf{S}(t)$, beyond the RWA limit, can be solved more expediently by the use of the MMFTGVV method and expressed in terms of three perturbed 
quasienergy eigenvalues and eigenvectors. That is, first we construct the density matrix operator $\hat{\rho}(t)$ according to the relation

$$
\hat{\rho}(t)=\widehat{U}(t ; 0) \hat{\rho}(0) \hat{U}^{\dagger}(t ; 0),
$$

where $\hat{U}(t ; 0)$ can be easily obtained from Eq. (42) or (43), and $\hat{\rho}(0)$ defines the initial state of the damping system.
The coherence vector $S_{i}(t), i=1,2, \ldots, 8$, can then be obtained directly by evaluating the traces defined in Eq. (72). Assuming the system is initially $(t=0)$ prepared in the ground level $|1\rangle$, and including the leading corrections due to the small perturbation $\widehat{V}_{3}$ defined in Eq. (50), the density matrix at large times $\left(t_{\infty} \gg 1 / g_{2}\right)$ can be obtained approximately analytically as

$$
\hat{\rho}\left(t_{\infty}\right)=e^{-2\left|\operatorname{Im}\left(\lambda_{1}\right)\right| t_{\infty}}\left[\begin{array}{ccc}
\frac{\beta^{4}}{\Omega^{4}}-\frac{4 \alpha \beta^{3} \Delta D}{\Omega^{8}} & \frac{\beta^{3} D}{\Omega^{6}} & \frac{-\alpha \beta^{3}}{\Omega^{4}}+\frac{Q}{\Omega^{8}} \\
\frac{\beta^{3} D}{\Omega^{6}} & 0 & \frac{-\alpha \beta^{2} D}{\Omega^{6}} \\
\frac{-\alpha \beta^{3}}{\Omega^{4}}+\frac{Q^{*}}{\Omega^{8}} & \frac{-\alpha \beta^{2} D}{\Omega^{6}} & \frac{\alpha^{2} \beta^{2}}{\Omega^{4}}+\frac{2 \alpha \beta\left(\alpha^{2}-\beta^{2}\right) \Delta D}{\Omega^{8}}
\end{array}\right],
$$

where

$$
\begin{aligned}
& D=\alpha \beta\left(-\delta_{1}+\delta+\delta_{3}\right)+\left(\alpha^{2}-\beta^{2}\right) \delta_{13}, \\
& Q=\beta^{2} D\left[\left(\alpha^{2}-\beta^{2}\right)\left(\Delta+i g_{2}\right)+2 \alpha^{2}\left(\Delta-i g_{2}\right)\right],
\end{aligned}
$$

and $\operatorname{Im}\left(\lambda_{1}\right)$ is given in Eq. (54). From Eq. (84), we see that at some large times $t_{\infty}$ the coherence vector components become

$$
\begin{aligned}
& S_{1}=S_{2}=0 \\
& S_{3}=\frac{4 \alpha \beta^{3}\left(\alpha^{2}-\beta^{2}\right) \Delta D}{\Omega^{10}} \exp \left[-2\left|\operatorname{Im}\left(\lambda_{1}\right)\right| t_{\infty}\right] \\
& S_{4}=\frac{-2 \beta^{2} D g_{2}}{\Omega^{6}} \exp \left[-2\left|\operatorname{Im}\left(\lambda_{1}\right)\right| t_{\infty}\right], \\
& S_{5}=\frac{2 \beta^{2} D}{\Omega^{5}} \exp \left[-2\left|\operatorname{Im}\left(\lambda_{1}\right)\right| t_{\infty}\right] \\
& S_{6}=0, \\
& S_{7}=\frac{-2 \beta^{2}\left(5 \alpha^{4}-2 \alpha^{2} \beta^{2}+\beta^{4}\right) \Delta D}{\Omega^{6}} \exp \left[-2\left|\operatorname{Im}\left(\lambda_{1}\right)\right| t_{\infty}\right]
\end{aligned}
$$

and

$$
\begin{aligned}
S_{8}= & \left(\frac{2 \beta^{2}}{\sqrt{3} \Omega^{2}}+\frac{4 \alpha^{3} \beta\left(\alpha^{2}-5 \beta^{2}\right) \Delta D}{\sqrt{3} \Omega^{10}}\right] \\
& \times \exp \left[-2\left|\operatorname{Im}\left(\lambda_{1}\right)\right| t_{\infty}\right] .
\end{aligned}
$$

Note that $S_{3}, S_{4}, S_{5}, S_{7}<<S_{8} \approx\left(2 \beta^{2} / \sqrt{3} \Omega^{2}\right) e^{-2\left|\operatorname{Im}\left(\lambda_{1}\right)\right| t_{\infty}}$. Thus at the two-photon resonance condition, allowing for the perturbations from non-RWA terms, the system reduces to a nearly coherent superposition state of levels 1 and 3. The eight-dimensional coherence vector evolves predominantly to a one-dimensional scalar and the population is quasitrapped mainly in the $S_{8}$ component as long as $t_{\infty} \gg 1 /\left|\operatorname{Im}\left(\lambda_{1}\right)\right|$. At further larger times
$\left|\operatorname{Im}\left(\lambda_{1}\right)\right| t_{\infty} \geq 1$, the system eventually completely decays away.

\section{A. Case study of SU(3) coherence vector dissipating}

The time-evolution of the coherent vector $\mathbf{S}(t)$ defined in Eq. (72) for the dissipative three-level $\Lambda$ system are shown, along with its nondamping counterpart for comparison, in this section. In Fig. 7 we present the first three components of $\mathbf{S}(t)$ as functions of time at the same conditions as those in Fig. 4(a). For the nondamping case, see Fig. 7(b), we have shown in a previous paper ${ }^{4}$ that these three components are intimately correlated and depict the one-photon coherence between two of the quasienergy states. For the dissipative system prescribed in the Sec. III C, these three components decay away rapidly as did the $P_{12}(t)$ in Fig. 4(a). The trajectory of $\mathbf{S}(t)$ projected onto the plane spanned by components $S_{1}(t)$ and $S_{2}(t)$, Fig. 8(a), showing a shrinking ellipse at a fast rate of the same order of magnitude as the loss rate $g_{2}$ which characterizes the second level. Figure $8(\mathrm{~b})$ indicates the stable ellipse for the nondamping case. We note here that the ellipse shown in Fig. 8(b) is calculated at the shifted, due to the antirotating effect, two-photon resonance defined at the maximum of the concaving curve in Fig. 3(a), instead of at the usual RWA two-photon resonance defined by the relation $\omega_{1}-\omega_{2}=\left|E_{3}-E_{1}\right|$.

In Fig. 9 we show the four components, which are closely correlated as seen in Eq. (80), of $\mathbf{S}(t)$, as functions of time. The character of the oscillations of these four components simulate those of $P_{11}(t)$ and $P_{13}(t)$ of Fig. $4(\mathrm{a})$ or $4(\mathrm{~b})$, except these component's converge oscillatorily to zero, and thus reveal the nature of two-photon coherence between the unperturbed levels 1 and 3. The two-photon coherence can be best visualized from the trajectory of the coherence vector $\mathbf{S}(t)$ projected onto the planes spanned by components $S_{4}(t)$ and $S_{7}(t)$ (Fig. 10), and $S_{5}(t)$ and $S_{6}(t)$ (Fig. 11). For the dissipative system, the trajectory on the $S_{5}-S_{6}$ plane, Fig. 11(a), becomes more and more confined toward the center, while for the 


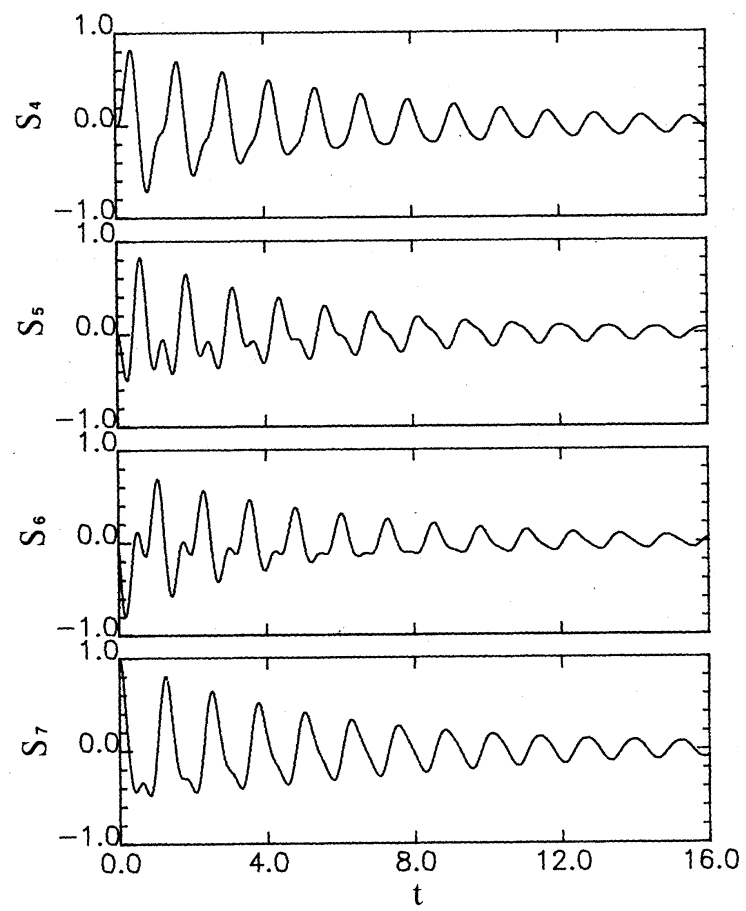

(a)

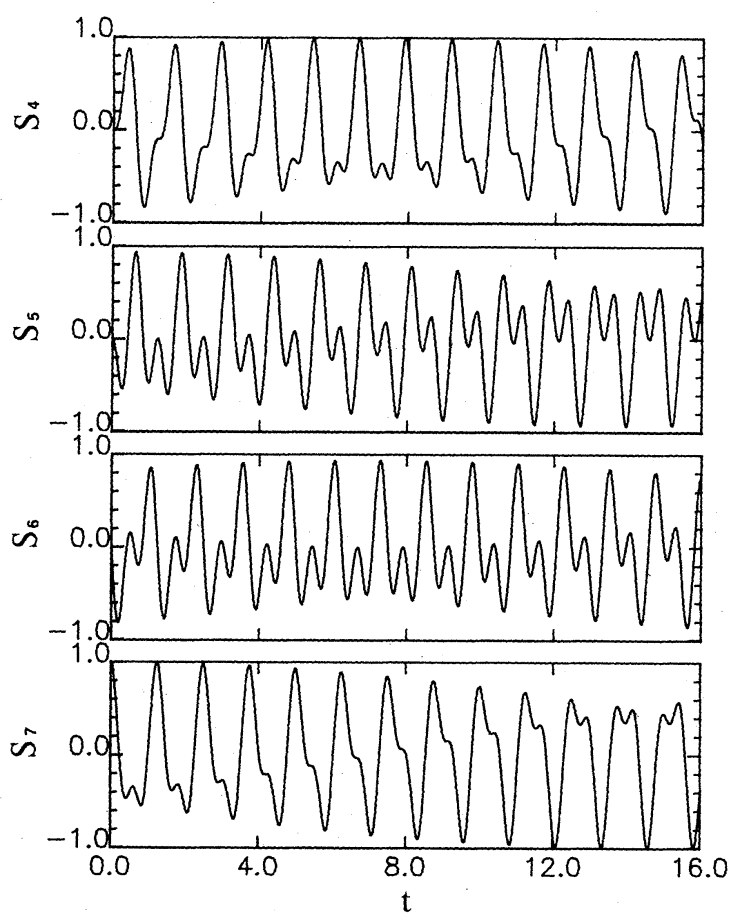

(b)

FIG. 9. Four components of the coherence vector, $S_{4}(t)$, $S_{5}(t), S_{6}(t)$, and $S_{7}(t)$, as functions of time for (a) the dissipative three-level system and (b) the nondamping system at the same conditions as depicted in Fig. 4.
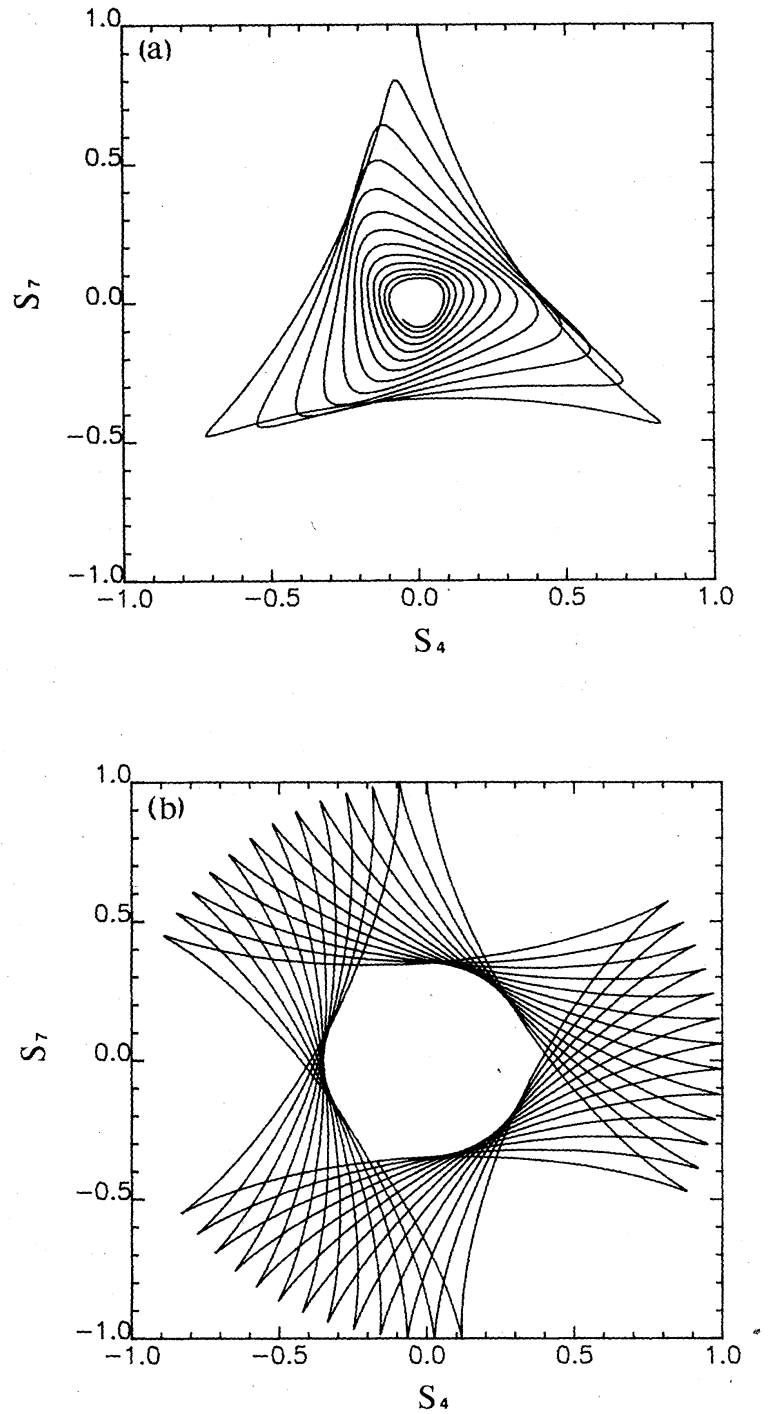

FIG. 10. Projection of the trajectory of the coherence vector on the $S_{4}-S_{7}$ plane for the same duration as shown in Fig. 9 for (a) the dissipative three-level system and (b) the nondamping system.

nondamping case, Figs. $10(\mathrm{~b})$ and $11(\mathrm{~b})$, the trajectory rotates with a constant amplitude. From Figs. 7-11, we have found that although all of the first seven components, i.e., $S_{1}, S_{2}, \ldots, S_{7}$ of $\mathbf{S}(t)$ vanish eventually at very large time, the four components, i.e., $S_{4}, S_{5}, S_{6}$, and $S_{7}$, which are closely related to the two-photon coherence between levels 1 and 3 persist more than the other three components, i.e., $S_{1}, S_{2}$, and $S_{3}$, which characterize the one-photon oscillation. One of the most striking features of the dissipative coherence vector $\mathbf{S}(t)$ is manifested through the eighth component $S_{8}(t)$. For the nondamping case, $S_{8}(t)$ evolves independently at the two-photon resonance (corrected by the antirotating effect), but for the dissipative case, Fig. 12, it grows with the time at first, then levels off, and eventually drops to zero at some very large time (not shown) despite its coupling with the com- 

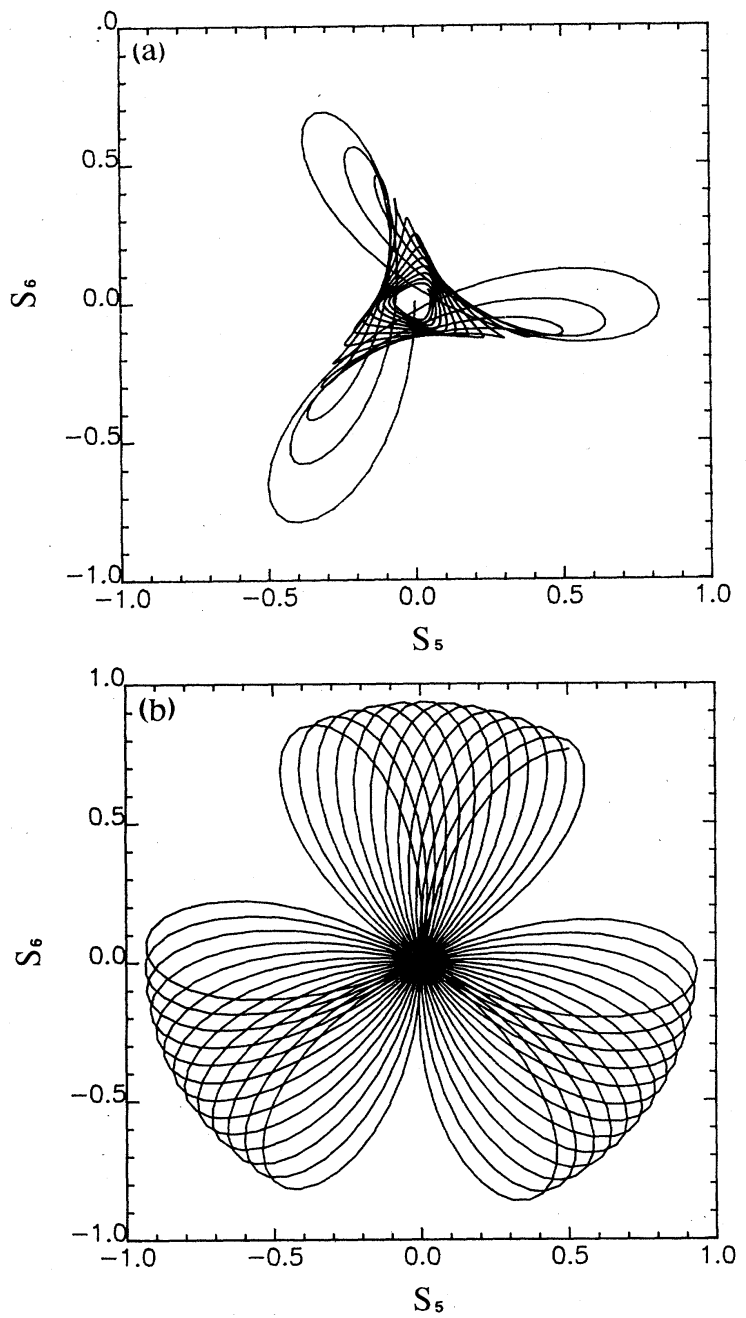

FIG. 11. Projection of the trajectory of the coherence vector on the $S_{5}-S_{6}$ plane for the same duration as shown in Fig. 9 for (a) the dissipative three-level system and (b) the nondamping system.

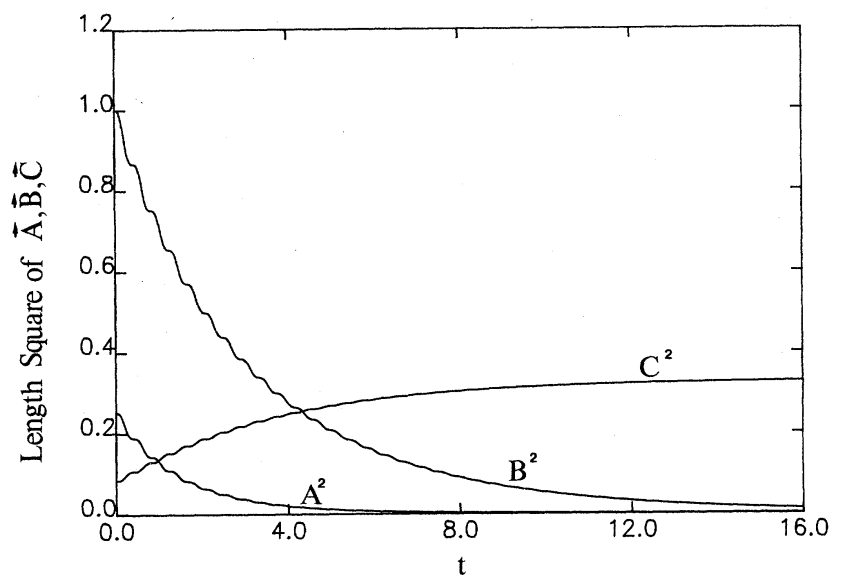

FIG. 12. The length square of the subvectors $\mathbf{A} \equiv\left(S_{1}(t), S_{2}(t), S_{3}(t)\right), \quad \mathbf{B}(t) \equiv\left(S_{4}(t), S_{5}(t), S_{6}(t), S_{7}(t)\right), \quad$ and $\mathrm{C} \equiv\left(S_{8}(t)\right)$ as functions of time for the same case as depicted in Fig. 4.

ponents $S_{1}, S_{2}$, and $S_{3}$, cf. Eq. (79), while the length of the other two subvectors, formed separately by $S_{1}, S_{2}, S_{5}$, $S_{6}$, and $S_{7}$, fall off quickly to zero. It is easily seen that this eighth component $S_{8}(t)$ is the manifestation of the quasitrapping experienced by the damping system.

\section{ACKNOWLEDGMENTS}

This work was supported in part by the U.S. Department of Energy (Division of Chemical Sciences), by the Alfred P. Sloan Foundation (S.-I C.), and by the University of Kansas-General Research Fund. Acknowledgment is also made to the Donors of the Petroleum Research Fund, administered by the American Chemical Society, for partial support of this work.
${ }^{1}$ For a recent review, see S.-I Chu, Adv. At. Mol. Phys. (to be published), and references therein.

${ }^{2}$ T.-S. Ho, S.-I Chu, and J. V. Tietz, Chem. Phys. Lett 96, 464 (1983).

${ }^{3}$ T.-S. Ho and S.-I Chu, J. Phys. B 17, 2101 (1984).

${ }^{4}$ T.-S. Ho and S.-I Chu, Phys. Rev. A 31, 659 (1985).

5 J. H. Shirley, Phys. Rev. 138, B979 (1965).

${ }^{6} J$. Elgin, Phys. Lett. 80A, 140 (1980).

${ }^{7}$ F. T. Hioe and J. H. Eberly, Phys. Rev. Lett. 47, 838 (1981); Phys. Rev. A 25, 2168 (1982).

${ }^{8}$ R. M. Whitley and C. R. Stroud, Jr., Phys. Rev. A 14, 1498 (1976); P. M. Radmore and P. L. Knight, J. Phys. B 15, 561 (1982).

${ }^{9}$ G. Orriols, Nuovo Cimento B 53, 1 (1979).

${ }^{10}$ B. J. Dalton and P. L. Knight, Opt. Commun. 42, 411 (1982); J. Phys. B 15, 3997 (1982); S. Swain, ibid. 15, 3405 (1982); T. A. B. Kennedy and S. Swain, ibid. 17, L389 (1984).

${ }^{11}$ G. Alzetta, A. Gozzini, L. Moi, and G. Orriols, Nuovo Cimento B 36, 5 (1976); G. Alzetta, L. Moi, and G. Orriols, ibid. 52, 209 (1979); J. E. Thomas, P. R. Hemmer, S. Ekezel,
C. C. Leiby, Jr., R. H. Picard, and C. R. Willis, Phys. Rev. Lett. 48, 867 (1982); H. R. Gray, R. M. Whitley, and C. R. Stroud, Jr., Opt. Lett. 3, 218 (1978).

${ }^{12}$ M. Kaivola, N. Bjerre, and O. Poulsen, Opt. Commun. 49, 418 (1984).

${ }^{13}$ R. P. Hackel and S. Ezekiel, Phys. Rev. Lett. 42, 1736 (1979); C. Delsart and J. C. Keller, J. Phys. (Paris) 39, 350 (1978); R. E. Tench, B. W. Peuse, P. R. Hammer, J. E. Thomas, S. Ezekiel, C. C. Leiby, Jr., R. H. Picard, and C. R. Willis, J. Phys. (Paris) Colloq. 42, C8-45 (1981); J. E. Thomas, S. Ezekiel, C. C. Leiby, Jr., R. H. Picard and C. R. Willis, Opt. Lett. 6, 298 (1981).

${ }^{14}$ S.-I Chu and W. P. Reinhardt, Phys. Rev. Lett. 39, 1195 (1977); S.-I Chu, Chem. Phys. Lett. 54, 367 (1978); A. Maquet, S.-I Chu, and W. P. Reinhardt, Phys. Rev. A 27, 2946 (1983).

${ }^{15}$ S.-I Chu, J. Chem. Phys. 75, 2215 (1981); S.-I Chu, C. Laughlin, and K. K. Datta, Chem. Phys. Lett. 98, 476 (1983).

16J. Aguilar and J. M. Combes, Comm. Math. Phys. 22, 265 (1971); E. Balslev and J. M. Combes, Commun. Math. Phys. 
22, 280 (1971); B. Simon, Ann. Math. 97, 247 (1973).

${ }^{17}$ W. P. Reinhardt, Annu. Rev. Phys. Chem. 33, 223 (1982); B. R. Junker, Adv. At. Mol. Phys. 18, 207 (1982).

${ }^{18}$ H. A. Yamani and W. P. Reinhardt, Phys. Rev. A 11, 1144 (1975).

${ }^{19}$ S.-I Chu (unpublished).

${ }^{20}$ M. P. Silverman and F. M. Pipkin, J. Phys. B 5, 1844 (1972);

D. R. Dion and J. O. Hirschfelder, Adv. Chem. Phys. 35, 265

(1976). In this latter paper the authors have discussed to some extent the introduction of phenomenological damping constants in the Floquet treatment of the interaction of twolevel systems with one monochromatic field.

${ }^{21}$ B. Kirtman, J. Chem. Phys. 49, 3890 (1968); 75, 798 (1981).

${ }^{22}$ P. R. Certain and J. O. Hirschfelder, J. Chem. Phys. 52, 5977 (1970); J. O. Hirschfelder, Chem. Phys. Lett. 54, 1 (1978); P. K. Aravind and J. O. Hirschfelder, J. Phys. Chem. 88, 4788 (1984).

${ }^{23}$ L. Mirsky, in An Introduction to Linear Algebra (Clarendon, Oxford, 1955), Chap. 7. 